\title{
Efficacy and safety of traditional Chinese medicine on treating oligomenorrhea: a systematic review and meta-analysis
}

\author{
Yannan $\mathrm{Li}^{1,2,3 \#}$, Guozhen Zhao, ${ }^{1,4 \#}$, Wei Shi ${ }^{3}$, Yin Zhang ${ }^{5}$, Hanlin Diao ${ }^{2,3}$, Nan Ding ${ }^{2,3}$, Panpan $\mathrm{Li}^{2,3}$, \\ Fang Zhang ${ }^{2,3}$, Lei Yang ${ }^{2,3}$, Mengyao Sun ${ }^{2,3}$, Hang Yu ${ }^{2,3}$, Bo Li ${ }^{6,7}, \mathrm{Li} \mathrm{Xu}^{8}$ \\ ${ }^{1}$ Second Clinical Medical College, Beijing University of Chinese Medicine, Beijing, China; ${ }^{2}$ School of Chinese Medicine, Shandong University \\ of Traditional Chinese Medicine, Jinan, China; ${ }^{3}$ Department of Gynecology, Affiliated Hospital of Shandong University of Chinese Medicine, \\ Jinan, China; ${ }^{4}$ Department of Emergency Medicine, Beijing Hospital of Traditional Chinese Medicine, Capital Medical University, Beijing, \\ China; ${ }^{5}$ Insititue of Basic Research in Clinical Medicine, China Academy of Chinese Medical Sciences, Beijing, China; ${ }^{6}$ Clinical Medical College \\ of Traditional Chinese Medicine, Capital Medical University, Beijing, China; ${ }^{7}$ Beijing Institute of Traditional Chinese Medicine, Beijing, China; \\ ${ }^{8}$ Department of Traditional Chinese Medicine, Shandong Provincial Hospital Affiliated to Shandong University, Jinan, China \\ Contributions: (I) Conception and design: W Shi, L Xu, Y Li, G Zhao; (II) Administrative support: W Shi; (III) Provision of study materials or \\ patients: Y Li, G Zhao; (IV) Collection and assembly of data: P Li, F Zhang; (V) Data analysis and interpretation: Y Li; (VI) Manuscript writing: All \\ authors; (VII) Final approval of manuscript: All authors. \\ "These authors contributed equally to this work and should be considered as co-first authors. \\ Correspondence to: Wei Shi. Department of Gynecology, Affiliated Hospital of Shandong University of Chinese Medicine, Lixia District, Jinan 250013, \\ China. Email: sw19781214@163.com; Li Xu. Department of Traditional Chinese Medicine, Shandong Provincial Hospital Affiliated to Shandong \\ University, Huaiyin District, Jinan 250021, China. Email: xulisw@126.com.
}

Background: Oligomenorrhea is one of the most frequent gynecologic complaints that interferes with women's life quality. Treatment using traditional Chinese medicine (TCM) is a preferred alternative therapy for oligomenorrhea. However, systematic reviews (SRs) evaluating the efficacy of TCM treatments for oligomenorrhea remain absent. The present SR and meta-analysis aimed to evaluate the efficacy and safety of TCM treatment for oligomenorrhea.

Methods: Randomized controlled trials (RCTs) published in English and Chinese were retrieved by searching in the databases in October 2019, including PubMed, Cochrane Library, EMBASE, Sinomed, CNKI, VIP, and WanFang databases. Quantitative analyses and quality assessments were then conducted based on abstracted data. This study protocol is registered PROSPERO, number CRD42018095660.

Results: A total of 26 eligible RCTs involving 2,389 patients were included in our analysis. Overall, we observed an effect of increasing menstrual blood volumes owing to using TCM treatments plus bio-medicine $(\mathrm{BM})\left(\mathrm{n}=649 ; \mathrm{MD}, 12.05 ; 95 \%\right.$ CI: 5.23 to $\left.18.87 ; \mathrm{P}<0.00001 ; \mathrm{I}^{2}=96 \%\right)$. Besides, TCM combined with $\mathrm{BM}$ yielded a significant prolongation in menstrual periods (MD, 1.20; 95\% CI: 0.78 to 1.62 ; $\mathrm{P}<0.00001$; $\mathrm{I}^{2}=76 \%$ ), and had potential improvements on enhancing effectiveness rates, increasing endometrial thickness, and raising the levels of estradiol $\left(\mathrm{E}_{2}\right)$ and progesterone $(\mathrm{P})$. Concerning adverse events (AEs), no significant difference was found in either group. The quality of evidence was relatively low.

Conclusions: This study seems to support the potential effect of TCM on treating oligomenorrhea. However, the relatively low quality of prior studies calls for future RCTs to further assess the efficacy of TCM on treating oligomenorrhea using rigorous designs.

Keywords: Chinese medicine therapy; oligomenorrhea; randomized controlled clinical trial; systematic evaluation; meta-analysis

Submitted Apr 07, 2020. Accepted for publication Dec 01, 2020.

doi: $10.21037 /$ apm-20-825

View this article at: http://dx.doi.org/10.21037/apm-20-825 


\section{Introduction}

Oligomenorrhea refers to an obvious reduction in menstrual blood, or a menstrual blood loss less than $30 \mathrm{~mL}$ per cycle, or a menstrual duration shorter than two days $(1,2)$. The prevalence of oligomenorrhea has been reported to be $10 \%$ to $15 \%$, which is considered as one of the most frequent gynecologic complaints that interfere with women's life quality $(3,4)$. Many factors can lead to oligomenorrhea, such as ovarian dysfunction (5), thyroid dysfunction (6), polycystic ovary syndrome (7), hyperprolactinemia (8), and the use of contraceptives (9). These factors can affect the secretion of gonadal hormones and the hypothalamus-pituitaryovary axis function. Surgical trauma and inflammationinduced abortion and intrauterine adhesion can damage the endometrium, resulting in thin endometrium and a decrease in estrogen and progesterone receptors (10-12). Also, stress, inappropriate weight loss, obesity, bad living habits can disturb the function of the hypothalamic-pituitary-ovarian axis and oligomenorrhea ensues $(13,14)$. Untimely treatments might lead to serious consequences such as amenorrhea, recurrent abortion, infertility, and premature ovarian failure. Hormonal therapy has been recommended as the first-line treatment for this condition. Hormone therapy, however, has a long course of treatment. It will increase the potential risks of cardiovascular system and metabolism and display a high recurrence rate after drug discontinuance. Moreover, evidence shows that patients receiving hormone therapy have low clinical acceptance and poor compliance (15-17).

Traditional Chinese medicine (TCM) has been widely used in China with a history of more than 2,000 years (18). TCM treatment, as one of the promising complementary therapies for oligomenorrhea, has been extensively explored and has shown great prospects in clinical practice. The main therapeutic principles of treating oligomenorrhea using TCM includes tonifying spleen and kidney, nourishing blood, and removing blood stasis, as well as soothing the liver. Many patients with oligomenorrhea opted for TCM treatments, because of the effective elimination of clinical symptoms and few adverse events (AEs) $(14,19)$. In the latest guidelines for diagnosis and treatment of abnormal uterine bleeding published by the Obstetrics and Gynecology Branch of the Chinese Medical Association (20), it is clearly proposed that patients with oligomenorrhea can be treated by a TCM therapy of activating blood. For oligomenorrhea patients only with thin endometrium, no definite treatment principle in bio-medicine (BM) has been proposed by far.
During that time, TCM treatment for oligomenorrhea is a preferred alternative therapy. However, no systematic review (SR) evaluating the efficacy of oligomenorrhea using TCM treatments has been reported in the literature. Therefore, we performed the present SR and meta-analysis to evaluate the quality of prior evidence supporting the efficacy and safety of TCM treatments for oligomenorrhea.

We present the following article in accordance with the PRISMA reporting checklist (available at http://dx.doi. org/10.21037/apm-20-825).

\section{Methods}

This study protocol is registered PROSPERO, number CRD42018095660. This review study was conducted based on the PRISMA statement (21).

\section{Search strategy}

We searched PubMed, Cochrane Library, EMBASE, Chinese Biomedical Literature Database (Sinomed), Chinese National Knowledge Infrastructure (CNKI), China Science and Technology Journal Database (VIP), and WanFang Data Knowledge Service Platform databases to identify eligible studies published from inception to October 30, 2019, with the language restriction of Chinese and English. We also manually searched the reference lists of the studies which were included to identify any other eligible publications. We used free words and MeSH terms including 'Oligomenorrhea', 'Oligomenorrheas' during conducting literature search, and the search strategy was shown in Figure S1. Two independent investigators (YNL and GZZ) undertook the estimation of eligible titles and abstracts firstly and then identified the original text of the relevant researches, then further read the full text of the screened articles, and finally identified the eligible researches that meet the inclusion criteria. When there are any disagreements, the consensus was reached through discussions with the third investigator (BL).

\section{Study selection}

We included randomized controlled trials (RCTs), comparing TCM combined with BM therapy versus BM alone or placebo plus BM. We reported the individuals of oligomenorrhea and the diagnosis for participants with 
oligomenorrhea following the definite or internationally acknowledged criteria. The studies were excluded, if the interventions reported were combined with other regimens such as acupuncture and acupoint injection. Duplicated literature, literature without relevant outcomes or failing to extract outcomes, and literature with apparently wrong randomization were also excluded. The primary outcomes were changes in menstrual blood volumes and menstrual periods, and AEs. The secondary outcomes included effectiveness rate, endometrial thickness, and the levels of follicle-stimulating hormone (FSH), luteinizing hormone $(\mathrm{LH})$, estradiol $\left(\mathrm{E}_{2}\right)$, and progesterone $(\mathrm{P})$.

\section{Data extraction}

Another two investigators (PPL and FZ) conducted the literature screening and data extraction independently and finally cross-checked. In cases of any disagreements, the consensus was reached through discussions with the third investigator (WS). Data extraction information included: titles of the article, the first author, years of publication.

\section{Quality assessment}

By using the Risk of Bias tool from the Cochrane Collaboration, we assessed the methodological quality of the included studies which were classified as "low risk", "high risk", or "unclear" (22).

\section{Assessment of the quality of the evidence}

We estimated the evidence quality of the present review study following the recommendation by the GRADE guideline using GRADE profiler software (23).

\section{Statistical analysis}

We analyzed the data and examined the publication bias by funnel plots using Review Manager 5.3. A randomeffect model was adopted to calculate the $95 \%$ confidence interval (CI) and risk ratio (RR) or mean difference (MD) of outcomes, because of the differences in the inclusion criteria, intervention, and comparison reported in prior studies. Heterogeneity was measured using the $\mathrm{I}^{2}$ test. A higher $\mathrm{I}^{2}$ score was provided to present greater inconsistency. Subgroup analysis will be used to explore potential sources of heterogeneity. When possible and appropriate, the potential subgroup will analyze the types of western medicine.

\section{Results}

A total of 6,703 articles were identified by our initial search, including 54 English articles and 6,648 Chinese ones. By searching for omissive studies, we acquired 1 additional record. Of all the included studies, 5,862 were checked, after 841 duplicates were removed. According to the inclusion criteria, we excluded 5,862 articles that were not following our inclusion criteria regarding Population, Intervention, Comparison, Outcomes (PICOs) after reading the abstracts. Finally, 26 RCTs were identified as eligible and were included in the SR after the full-text reading was conducted. The PRISMA template (21) for study selection is displayed in Figure 1.

\section{Characteristics of the included trials}

Table 1 demonstrates the characteristics of the studies included in our analysis. All RCTs were conducted in China and were published in Chinese, with their sample size ranging from 50 to $242(25,29)$. The participant's ages ranged from 16 to 47 years old $(29,30)$. The course lengths of treatment varied from 4 weeks to 4 menstrual cycles, and most of them lasted 3 menstrual cycles. All studies used blank controls. The ingredients of the included Chinese herbal medicines are listed in Table S1.

\section{Methodological quality assessment}

By the Risk of Bias tool (22), overviews of the judgment with regards to the risk of bias items in the included studies were shown in Figure 2A,2B. Five trials reported the use of a random number table $(33,37,49)$ or computer randomization $(24,32)$ to generate random sequences, while the remaining 11 trials provided random allocation without any details. For all these trials, an unclear risk of bias in allocation concealment existed, and none of them reported the blinding of study personnel and participants. For items of "blinding of outcome assessment", "incomplete outcome data", "selective reporting", and "other bias", all included studies were assessed for low risk of bias regarding the outcome of "selective reporting", except for 2 studies $(24,40)$ in which the outcome of "selective reporting" was assessed for high risk of bias. 


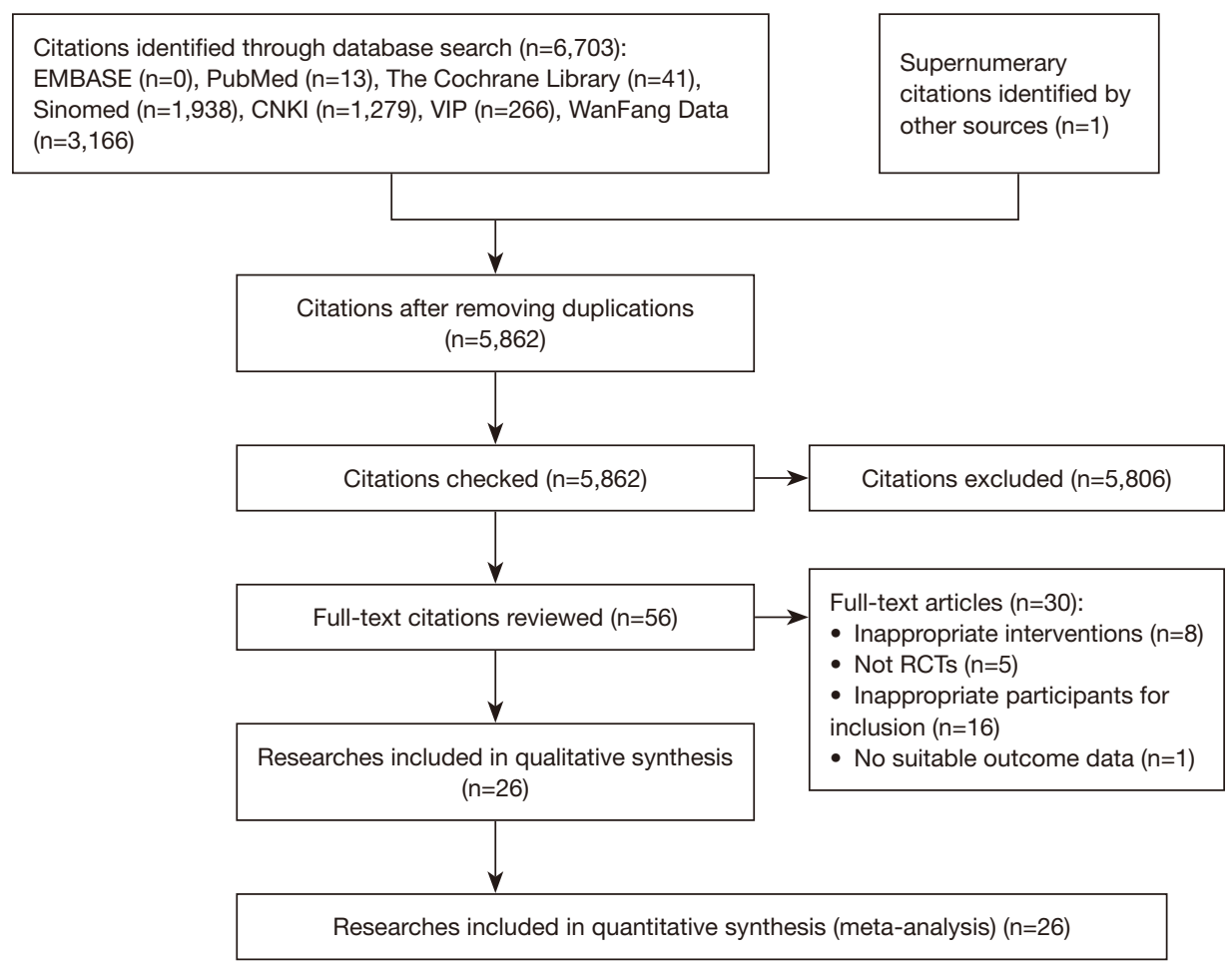

Figure 1 Flow diagram of study selection.

\section{Overall analyses}

\section{Primary outcomes}

\section{Changes in menstrual blood volume}

The changes in menstrual blood volume are shown in Figure 3. Of the included studies, 5 studies $(29,32,43,45,49)$ including 739 patients (364 in the treatment group versus 375 in the control group) reported the changes in menstrual blood volume. One study (32) used pictorial blood loss assessment chart (PBAC) to assess the menstrual blood volume. The result revealed a statistical difference between treatment and control groups $(\mathrm{n}=90 ; \mathrm{MD}, 9.51 ; 95 \% \mathrm{CI}$, 4.16 to $14.86, \mathrm{P}=0.0005)$. Another 4 studies $(29,43,45,49)$ including 649 patients (319 in the treatment group versus 330 in the control group) reported the specific menstrual blood volume and showed an effect of increasing menstrual blood volume due to using TCM treatments plus BM ( $\mathrm{n}=649 ; \mathrm{MD}$, 12.05; 95\% CI: 5.23 to 18.87 ; $\mathrm{P}<0.00001 ; \mathrm{I}^{2}=96 \%$ ). Subgroup analysis was performed for different bio-medicine regimens in the control group. One study (29) showed the increase of menstrual blood volume. It suggested that TCM + estradiol valerate + progesterone was better than estradiol valerate + progesterone ( $\mathrm{n}=242$; MD, 24.16; 95\% CI, 20.82 to 27.50, $\mathrm{P}<0.00001)$. Three studies $(43,45,49)$ assessed the increase of menstrual blood volume with TCM + estradiol valerate + cyproterone acetate versus estradiol valerate + cyproterone acetate with significant differences $(\mathrm{n}=407$; MD, 8.21; 95\% CI, 5.40 to $11.02 ; \mathrm{P}<0.00001 ; \mathrm{I}^{2}=74 \%$ ).

\section{Changes in menstrual period}

The comparison of menstrual periods is shown in Figure 4. Three studies $(25,29,42)$ involving 358 patients were analyzed. Compared with BM alone, TCM plus BM had statistical significance on prolonging the menstrual period that terminated prematurely (MD, $1.20 ; 95 \% \mathrm{CI}, 0.78$ to 1.62; $\left.\mathrm{P}<0.00001 ; \mathrm{I}^{2}=76 \%\right)$.

\section{Adverse events (AEs)}

Of the 8 studies $(24,28,33,34,39,43,44,49)$ that reported AEs, one (34) reported no AE and 7 studies $(24,28,33,39,43,44,49)$ described AEs (as shown in Figure 5). Pooled estimates of 7 studies investigated that there was no statistical significance in the frequency of AEs between the groups of TCM + BM and $\mathrm{BM}$ alone. These studies exhibited insignificant heterogeneity (RR, 0.61; 95\% CI, 0.37 to $1.01 ; \mathrm{P}=0.05 ; \mathrm{I}^{2}=24 \%$ ). Five types of AEs were reported in 7 studies that compared TCM + BM with BM. No significance between treatment and control groups was found. Regarding individual AEs, the most frequent $\mathrm{AE}$ was breast distension and pain in each group (as 
Table 1 Characteristics of the included studies

\begin{tabular}{|c|c|c|c|c|c|c|c|}
\hline \multirow{2}{*}{ Study ID } & \multirow{2}{*}{ Locations } & \multirow{2}{*}{$\begin{array}{c}\text { Sample size } \\
(T / C)\end{array}$} & \multirow{2}{*}{ Age (T/C, years) } & \multicolumn{2}{|c|}{ Treatment group/control group } & \multirow{2}{*}{ Course of treatment } & \multirow{2}{*}{ Outcomes } \\
\hline & & & & $T$ & c & & \\
\hline Cai C 2018 (24) & China & $25 / 26$ & Т: $30.26 \pm 6.69 ;$ C: $30.18 \pm 6.51$ & Bushen Yangjing decoction + BM & BM: Bromocriptine & 3 months & (3)(5)(6)(7)(9) \\
\hline Cheng H 2016 (25) & China & 30/20 & 19-41 & Yangijing Tongluo decoction + Hexue decoction + BM & BM: Estradiol valerate + Progesterone & 4 menstrual cycles & (2) (3) (4) 5)(6)(7)(8) \\
\hline Gao S 2014 (26) & China & $53 / 53$ & T: 20-45; C: 21-43 & Shenghua decoction + BM & BM: Estradiol valerate + Medroxyprogesterone & 3 months & (3) \\
\hline Hei L 2017 (27) & China & $43 / 43$ & Т: $30.26 \pm 6.69 ;$ C: $30.18 \pm 6.51$ & Yangshen Huayu decoction + BM & BM: Estradiol valerate + Progesterone & 3 months & (4) \\
\hline HuX 2017 (28) & China & $40 / 40$ & Т: $26.35 \pm 3.11 ;$ C: $26.26 \pm 3.07$ & Guishen decoction + BM & BM: Estradiol valerate + Medroxyprogesterone & 3 menstrual cycles & (4)(5)(6)(7)(8)(9) \\
\hline Li J 2018 (29) & China & $127 / 115$ & T: 18-45; C: 18-47 & Dingkun Dan + BM & BM: Estradiol valerate + Progesterone & 4 menstrual cycles & (1)(2)(3)(4)(5)(6)(7)(8) \\
\hline LiY 2012 (30) & China & $75 / 75$ & $16-43$ & Self-made decoction + BM & BM: Diethylstilbestrol + Progesterone & 3-6 menstrual cycles & (3) \\
\hline Lu K 2017 (32) & China & $45 / 45$ & Т: $30.13 \pm 4.51 ;$ C: $31.97 \pm 5.08$ & Maixuekang capsule + BM & BM: Estradiol valerate + Progesterone & 3 menstrual cycles & (1)(4) \\
\hline Lu Y 2016 (33) & China & 63/63 & Т: $24.5 \pm 5.7 ;$ C: $24.5 \pm 5.5$ & Self-made decoction + BM & BM: Conjugated estrogens + Cyproterone acetate & 3 months & (3)(5)(6)(7) (9) \\
\hline Ma X 2017 (34) & China & $25 / 25$ & 21-39 & Bushen Huoxue decoction + BM & BM: Estradiol valerate + Cyproterone acetate & 3 menstrual cycles & (3)(5)(7) \\
\hline Ruan R 2012 (35) & China & $30 / 30$ & $21-40$ & Jianpi Bushen Yijing Yangxue decoction + BM & BM: Estradiol valerate + Progesterone & 3 menstrual cycles & (3) \\
\hline Song J 2011 (36) & China & $30 / 30$ & Т: $26.67 \pm 4.47 ;$ C: $25.46 \pm 3.84$ & Kunling pill + BM & BM: Estradiol valerate + Cyproterone acetate & 3 menstrual cycles & (3)(4) \\
\hline Tang C 2018 (37) & China & $38 / 39$ & Т: $26.67 \pm 4.47 ;$ C: $25.46 \pm 3.84$ & Bushen Jianpi Yangxue electuary + BM & BM: Estradiol valerate + Progesterone & 3 menstrual cycles & (3)(4)(5)(6)(7)(8) \\
\hline Tian B 2016 (38) & China & $30 / 30$ & T: $27.7 \pm 2.1 ; \mathrm{C}: 27.4 \pm 2.2$ & Bushen Zixue decoction + BM & BM: Estradiol valerate + Cyproterone acetate & 3 menstrual cycles & (3)(4) \\
\hline Wang Q 2014 (39) & China & $28 / 28$ & Т: $26.57 \pm 3.58 ;$ C: $26.35 \pm 3.66$ & Siwu decoction + BM & BM: Estradiol valerate + Progesterone & - & (3)(9) \\
\hline Wang Y 2016 (40) & China & $30 / 30$ & Т: $30.90 \pm 6.11 ;$ C: $29.53 \pm 6.21$ & Yangjing Zixue decoction + BM & BM: Estradiol valerate & - & (3)(4) \\
\hline Xiong J 2017 (42) & China & $33 / 33$ & Т: $30.78 \pm 5.08 ;$ C: $32.23 \pm 4.28$ & Bushen Huoxue decoction + BM & BM: Estradiol valerate + Progesterone & 4 weeks & (2)(3)(4) \\
\hline Xue X 2015 (43) & China & $111 / 110$ & $28.66 \pm 4.39$ & Huoxue Tiaojing pill + BM & BM: Estradiol valerate + Cyproterone acetate & 3 menstrual cycles & (1)(3)(9) \\
\hline Zhang F 2015 (44) & China & $88 / 85$ & T: $27.4 \pm 8.9 ;$ C: $26.7 \pm 5.9$ & Chanfukang granule + BM & BM: Estradiol valerate + Progesterone & 3 menstrual cycles & (3)(9) \\
\hline Zhang H 2018 (45) & China & 30/30 & Т: $27.03 \pm 3.77 ;$ C: $29.40 \pm 4.93$ & Self-made decoction + BM & BM: Estradiol valerate + Cyproterone acetate & 3 menstrual cycles & (1)(3)(5)(6)(7) \\
\hline Zheng Y 2015 (46) & China & 30/30 & Т: $28.85 \pm 5.12 ;$ C: $29.45 \pm 4.93$ & Zishen Yutai pill + BM & BM: Estradiol valerate + Progesterone & 3 menstrual cycles & (3)(4) \\
\hline Zhang Z 2016 (47) & China & $43 / 43$ & Т: $32.8 \pm 2.4 ;$ C: $32.9 \pm 2.3$ & Bushen Jianpi Yiling Yangxue decoction + BM & BM: Estradiol valerate + Progesterone & 3 menstrual cycles & (3)(4) \\
\hline Zhao J 2014 (48) & China & $37 / 37$ & $28 \pm 5.2$ & Bushen Jianpi Yiling Yangxue decoction + BM & BM: Estradiol valerate + Progesterone & 3 menstrual cycles & (3)(4) \\
\hline Zheng Z 2015 (49) & China & $63 / 63$ & Т: $34.19 \pm 9.58 ;$ C: $35.33 \pm 9.31$ & Huoxue Tiaojing pill + BM & BM: Estradiol valerate + Cyproterone acetate & 3 menstrual cycles & (1) (3) (5)(6)(7)(8)(9) \\
\hline
\end{tabular}


A

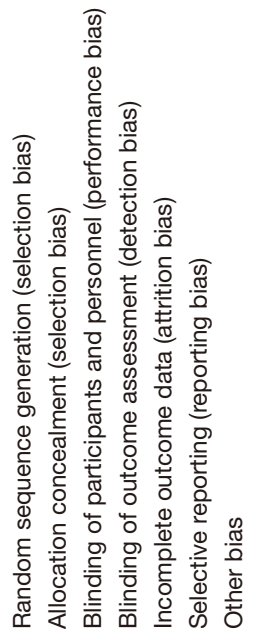

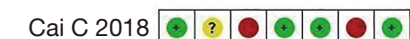

Cheng H 2016 ?

Gao S 2014 ?

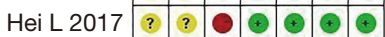

$\mathrm{HuX} 2017$ ?

Li J 2018 ?

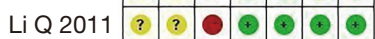

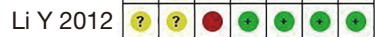

Lu K 2017 ๑ (-

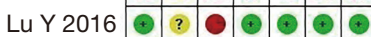

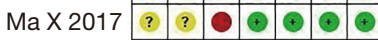

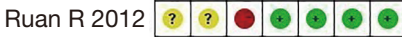

Song J 2011 ?

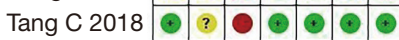

Tian B 2016 ?

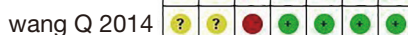

Wang Y 2016 ?

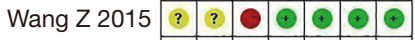

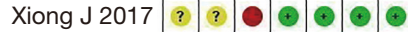

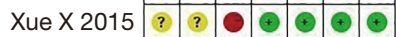

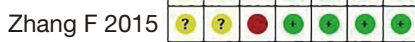

Zhang H 2018 ?

Zhang Z 2016 ?

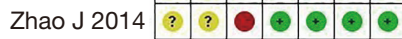

Zheng Y 2015 ?

Zheng Z 2015

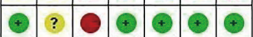

B Random sequence generation (selection bias)

Allocation concealment (selection bias)

Blinding of participants and personnel (performance bias)

Blinding of outcome assessment (detection bias)

Incomplete outcome data (attrition bias)

Selective reporting (reporting bias)

Other

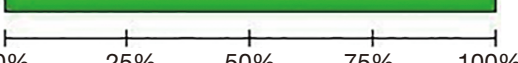

Low risk of bias

$\square$ Unclear risk of bias

$\square$ High risk of bias

Figure 2 Assessment of risk of bias (A) Risk of bias graph shows the risk of bias items of the included studies. (B) Risk of bias summary shows the risk of bias items of the included studies. 


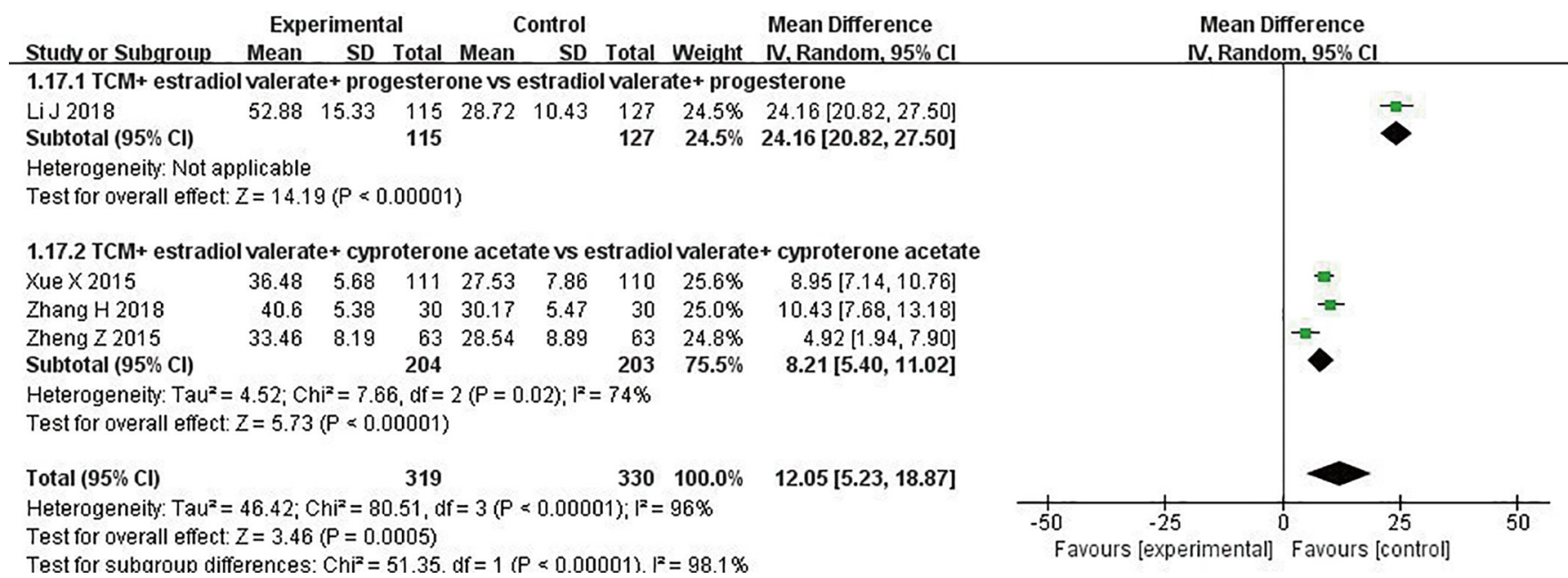

Figure 3 Changes in menstrual blood volume comparison.

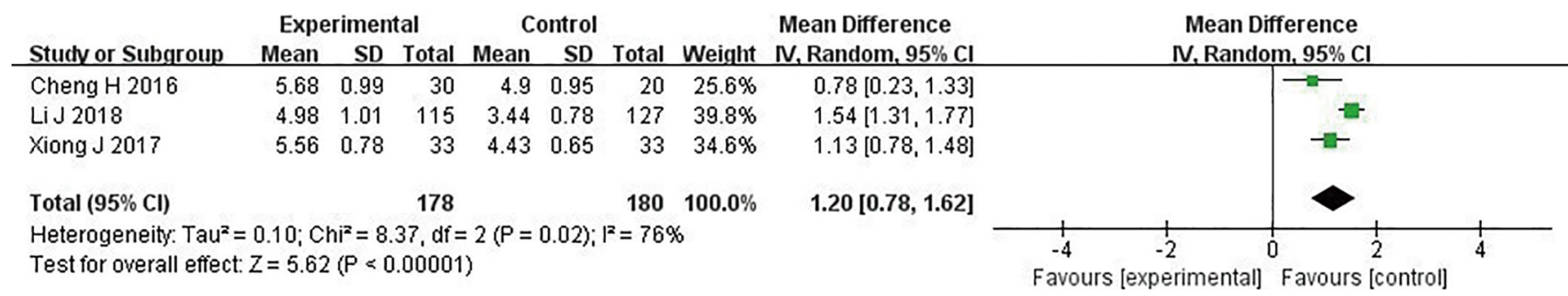

Figure 4 Changes in menstrual period comparison.

shown in Table 2). In the same light, there was no significance in routine blood and urine tests, and tests of liver and kidney functions before and after treatment.

\section{Secondary outcomes \\ Effectiveness rate}

Of the included studies, 25 studies (24-31,33-49) including 2,299 patients $(1,152$ in the treatment group and 1,147 in the control group) reported the effectiveness rate. Although some differences existed in the composition and dosage of medicines, meta-analysis showed that TCM treatments plus BM were of great benefits for the enhancement of effectiveness rates in oligomenorrhea patients compared with $\mathrm{BM}$ alone (RR, $1.23 ; 95 \% \mathrm{CI}, 1.18$ to 1.28 ; $\left.\mathrm{P}<0.00001 ; \mathrm{I}^{2}=2 \%\right)$. There was no statistical significance in heterogeneity among the studies previously mentioned (as shown in Figure S2).

\section{Endometrial thickness}

A total of 14 available RCTs $(25,27-29,32,36-38,37,40-42,46-48)$ showed the apparent effect on endometrial thickness.
Subgroup analyses were performed using TCM and $\mathrm{BM}$ to compare the endometrial thickness. Nine studies $(25,27,29,32,37,42,46-48)$ reported the increase of endometrial thickness. The results indicated that TCM + estradiol valerate + progesterone was better than estradiol valerate + progesterone $(\mathrm{n}=831 ; \mathrm{MD}, 1.01 ; 95 \% \mathrm{CI}, 0.47$ to $\left.1.55, \mathrm{P}=0.0002 ; \mathrm{I}^{2}=92 \%\right)$. Three studies $(36,38,41)$ assessed the increase of endometrial thickness using TCM + estradiol valerate + cyproterone acetate compared with estradiol valerate + cyproterone acetate. Significant differences were found between the two groups ( $\mathrm{n}=180 ; \mathrm{MD}, 1.46 ; 95 \% \mathrm{CI}$, 0.36 to $2.57 ; \mathrm{P}=0.009 ; \mathrm{I}^{2}=94 \%$ ). One study (40) assessed the increase of endometrial thickness using TCM + estradiol valerate compared with estradiol valerate with statistical significance between the two groups ( $\mathrm{n}=60 ; \mathrm{MD}, 0.60$; $95 \%$ CI, 0.02 to $1.18 ; \mathrm{P}=0.04)$. One study (28) assessed the increase of endometrial thickness using TCM + estradiol valerate + medroxyprogesterone compared with estradiol valerate + medroxyprogesterone with a statistical difference between the two groups $(\mathrm{n}=80 ; \mathrm{MD}, 1.19 ; 95 \% \mathrm{CI}, 0.77$ to 


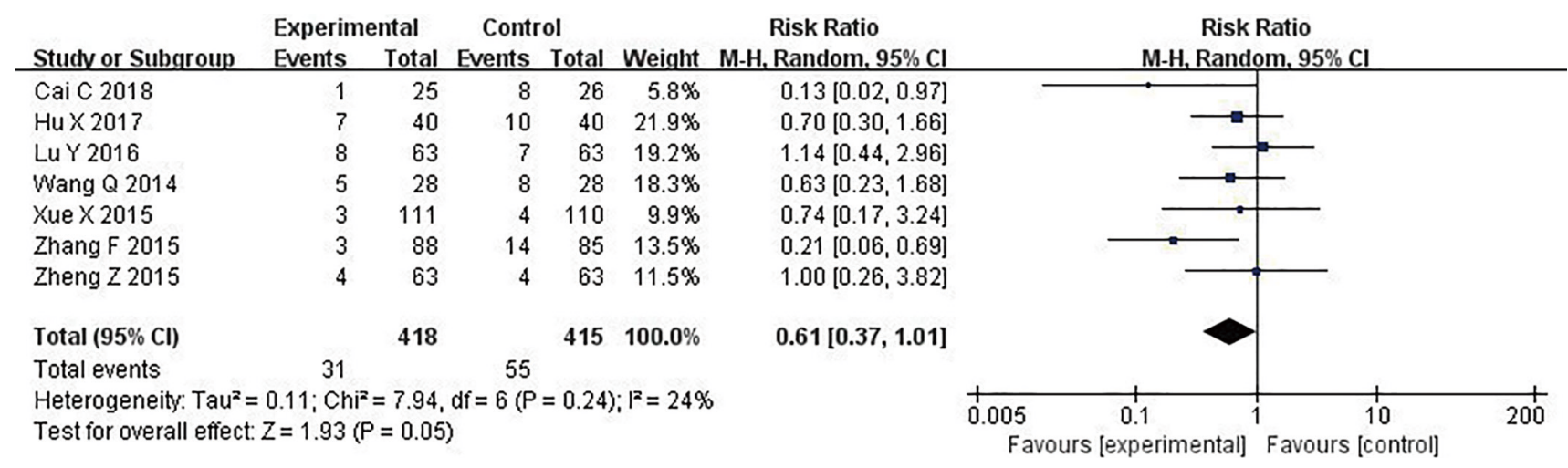

Figure 5 Adverse events comparison.

Table 2 Incidence of adverse events

\begin{tabular}{|c|c|c|c|c|c|c|}
\hline Adverse events & \multicolumn{2}{|c|}{ Total events/total number } & \multicolumn{2}{|c|}{ Heterogeneity test } & \multicolumn{2}{|c|}{ Meta-analysis } \\
\hline Nausea and vomiting & $6 / 353$ & $10 / 349$ & 0.72 & $0 \%$ & $0.75(0.26,2.12)$ & 0.59 \\
\hline Dizziness and headache & $7 / 365$ & $10 / 361$ & 0.84 & $0 \%$ & $0.71(0.27,1.84)$ & 0.48 \\
\hline Rash & $3 / 214$ & $4 / 213$ & 0.61 & $0 \%$ & $0.77(0.20,3.06)$ & 0.72 \\
\hline Gastrointestinal reactions & $5 / 131$ & $10 / 131$ & 0.91 & $0 \%$ & $0.50(0.18,1.42)$ & 0.19 \\
\hline
\end{tabular}

$\mathrm{TCM}+\mathrm{BM}$, traditional Chinese medicine + bio-medicine.

\subsection{1; $\mathrm{P}<0.00001$ ) (as shown in Figure $\mathrm{S} 3$ ).}

\section{Changes in hormone levels}

The changes of $\mathrm{E}_{2}, \mathrm{P}, \mathrm{FSH}$, and $\mathrm{LH}$ were displayed in Figures S4-S7. Individuals who were treated by TCM plus $\mathrm{BM}$ had a mean increase in $\mathrm{E}_{2}(24,25,28,29,33,34,37,45,49)$ $(\mathrm{n}=862 ; \mathrm{MD}, 5.19 ; 95 \% \mathrm{CI}, 2.06$ to $8.33 ; \mathrm{P}=0.001$; $\left.\mathrm{I}^{2}=97 \%\right)$, and $\mathrm{P}(25,28,29,37,49)(\mathrm{n}=575 ; \mathrm{MD}, 0.27 ; 95 \%$ CI, 0.07 to $\left.0.46 ; \mathrm{P}=0.007 ; \mathrm{I}^{2}=86 \%\right)$. Statistical significance was found between the two groups. FSH levels were reported in 9 studies $(24,25,28,29,33,34,37,45,49)$. A meta-analysis compared TCM + BM with BM alone failed to report significant differences in the changes in FSH ( $\mathrm{n}=862 ; \mathrm{MD},-0.09 ; 95 \% \mathrm{CI},-0.23$ to $0.04 ; \mathrm{P}=0.18$; $\left.\mathrm{I}^{2}=86 \%\right)$. Outcomes of $\mathrm{LH}$ were determined in 8 studies $(24,25,28,29,33,37,45,49)$. According to the results of metaanalyses, there was no significant difference in the levels of LH between the two groups ( $\mathrm{n}=812 ; \mathrm{MD}, 0.45 ; 95 \%$ CI, -0.12 to $\left.1.03 ; \mathrm{P}=0.12 ; \mathrm{I}^{2}=92 \%\right)$.

\section{Assessment of publication bias}

Publication bias regarding the effectiveness rate and the endometrial thickness was assessed using funnel plots. The result indicated that there was no significant publication bias in the effectiveness rate (as shown in Figure $6 A$ ), but great differences in endometrial thickness (Figure $6 B$ ).

\section{Quality of the evidence assessment}

In Table 3, we summarized the overall evidence for various outcomes (except for AEs), using the GRADE method. Generally, the quality of the evidence was "low" for the effect of TCM on effectiveness rates and was "very low" for the effects of TCM on changes in menstrual blood volumes, menstrual periods, endometrial thickness, and $\mathrm{E}_{2}, \mathrm{P}, \mathrm{FSH}$, and LH levels.

\section{Discussion}

Our results indicate that treatments of TCM combined with BM have better effects in treating oligomenorrhea, compared with BM alone. Specifically, we observed an effect of increasing menstrual blood volumes, prolonging menstrual periods, increasing endometrial thickness, and 
A

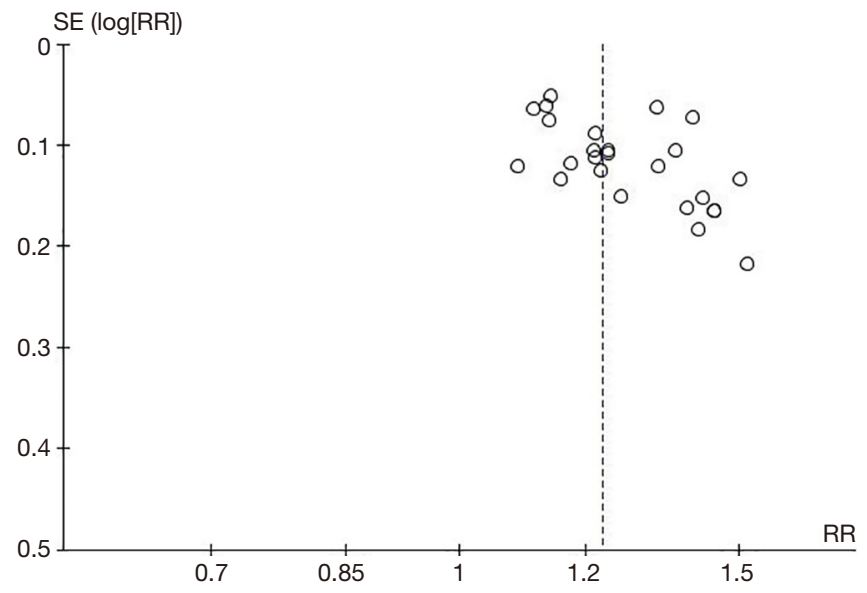

B

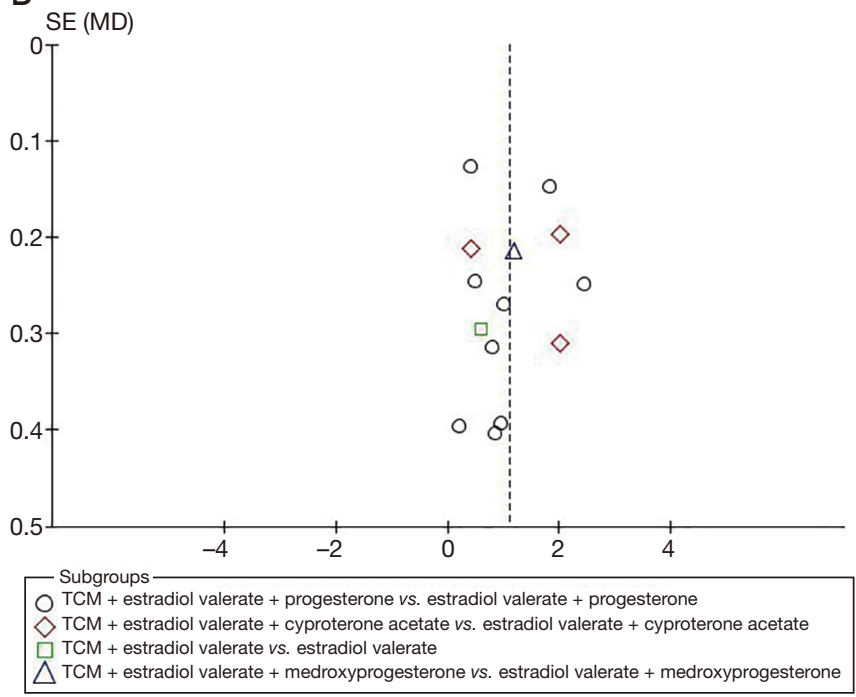

Figure 6 Assessment of publication bias. (A) Funnel plot of effective rate indicated that there was no significant publication bias in the effectiveness rate. (B) Funnel plot of endometrial thickness indicated that there was a publication bias in endometrial.

increasing the levels of estradiol $\mathrm{E}_{2}$ and $\mathrm{P}$ by using TCM treatments plus BM. Nevertheless, based on the result of the Cochrane Risk of Bias tool, high risk and unclear risk of bias were found, and the GRADE evidence displayed poor quality. The pooled estimates of included trials showed that there was no statistical significance in AEs. Besides, no acute $\mathrm{AE}$ was reported. In a word, TCM treatment for oligomenorrhea may be an alternative therapy with potential effectiveness and satisfying safety, it provides intriguing implications for clinical practice.

The mechanisms of TCM in treating oligomenorrhea remains partially clear. Based on specific TCM herbal prescriptions, the mechanisms may include phytoestrogenslike effects, anti-inflammatory effects, and improvement of blood supply, and so on. Prior studies have proved that Radix Angelicae Sinensis, Radix Astragali, and Folium Epimedii displayed an activity of estrogen regulation and can restore the number of follicles in the ovaries, thicken the uterine wall and increase the endometrium gland in proliferative or secretory phases on aged rats (50). An experiment has illustrated that Radix Paeoniae Alba can trigger phytoestrogens-like activities via increasing the expression of estrogen receptors in uterus and vagina and increasing serum estrogen level in mice with no AEs (51). Studies have shown that Radix Paeoniae Alba and Rhizoma Atractylodis Macrocephalae have the effect of suppressing the inflammatory response by inhibiting MAPK, NF- $\kappa \mathrm{B}$, and
PI3K/Akt signaling pathways (52,53). Animal experiments have demonstrated that in addition to promoting the expression of Th1 and Th2 cytokines in T lymphocytes and enhancing the immune function of rats, the components of Radix Rehmanniae Preparata can significantly promote the proliferation of bone marrow cells, increase the number of hemoglobin and erythrocyte, and improve the recovery process of hematopoietic functions $(54,55)$. Previous investigations have proved that chemical constituents of Semen Cuscutae can help to restore the function of hypothalamic-pituitary-gonads, promote the development of follicles, and enhance the number and function of ovarian hormone receptors (56-59). Rhizoma Chuanxiong, Radix Paeoniae Rubra, Radix Salviae Miltiorrhizae, Flos Carthami, and Hirudo are all classic Chinese medicines for promoting blood circulation and removing blood stasis in China, which have been considered effective in improving blood hemorheology, anti-platelet aggregation, antioxidant, antiapoptosis, and anti-inflammatory (60-63). Besides, some of the TCMs have been proved to ameliorate liver and kidney injury and have protective effects on the liver and kidney (64-67). In summary, these findings are consistent with the result of our study. There may be multiple mechanisms of TCM in treating oligomenorrhea, which are worthy of further research.

A previous SR showed that some medicinal herbs of Traditional Persian medicine were effective for the 
Table 3 Summary of the evidence for outcomes

\begin{tabular}{|c|c|c|c|c|c|c|c|c|c|c|c|c|}
\hline \multicolumn{7}{|c|}{ Quality assessment } & \multicolumn{2}{|c|}{ No of patients } & \multicolumn{2}{|r|}{ Effect } & \multirow[b]{2}{*}{ Quality } & \multirow[b]{2}{*}{ Importance } \\
\hline No. of studies & Design & Risk of bias & Inconsistency & Indirectness & Imprecision & $\begin{array}{c}\text { Other } \\
\text { considerations }\end{array}$ & $\begin{array}{l}\text { Traditional Chinese } \\
\text { medicine }\end{array}$ & Control & Relative $(95 \% \mathrm{Cl})$ & Absolute & & \\
\hline \multicolumn{13}{|c|}{ Changes in menstrual blood volume (follow-up mean 3 months; better indicated by lower values) } \\
\hline 4 & Randomized trial & Very serious ${ }^{1}$ & Serious $^{2}$ & $\begin{array}{l}\text { No serious } \\
\text { indirectness }\end{array}$ & Serious $^{3}$ & Reporting bias $^{4}$ & 319 & 330 & - & MD 12.05 higher (5.23 to 18.87 higher) & $\begin{array}{c}\text { o++ooo Very } \\
\text { low }\end{array}$ & Critical \\
\hline \multicolumn{13}{|c|}{ Changes in menstrual period (follow-up mean 3 months; better indicated by lower values) } \\
\hline 3 & Randomized trial & Very serious & Serious & $\begin{array}{l}\text { No serious } \\
\text { indirectness }\end{array}$ & Serious & Reporting bias & 178 & 180 & - & MD 1.2 higher (0.78 to 1.62 higher) & $\begin{array}{c}\text { o++ooo Very } \\
\text { low }\end{array}$ & Critical \\
\hline \multicolumn{13}{|c|}{ Effective rate (follow-up mean 3 months) } \\
\hline 25 & Randomized trial & Very serious & $\begin{array}{l}\text { No serious } \\
\text { inconsistency }\end{array}$ & $\begin{array}{l}\text { No serious } \\
\text { indirectness }\end{array}$ & $\begin{array}{l}\text { No serious } \\
\text { imprecision }\end{array}$ & None & 1,048/1,152(91\%) & $\begin{array}{c}825 / 1,147(71.9 \%) \\
73 \%\end{array}$ & RR $1.23(1.18$ to 1.28$)$ & $\begin{array}{l}165 \text { more per } 1,000 \text { (from } 129 \text { more to } 201 \text { more) } \\
168 \text { more per } 1000 \text { (from } 131 \text { more to } 204 \text { more) }\end{array}$ & ०++++++০ Low & Critical \\
\hline \multicolumn{13}{|c|}{ Endometrial thickness (follow-up mean 3 months; better indicated by lower values) } \\
\hline 14 & Randomized trial & Very serious & Serious & $\begin{array}{l}\text { No serious } \\
\text { indirectness }\end{array}$ & $\begin{array}{l}\text { No serious } \\
\text { imprecision }\end{array}$ & Reporting bias & 574 & 577 & - & MD 1.1 higher ( 0.69 to 1.5 higher) & $\begin{array}{l}\text { o++ooo Very } \\
\text { low }\end{array}$ & Important \\
\hline \multicolumn{13}{|c|}{ LH (follow-up mean 3 months; better indicated by lower values) } \\
\hline 8 & Randomized trial & Very serious & Serious & $\begin{array}{l}\text { No serious } \\
\text { indirectness }\end{array}$ & $\begin{array}{l}\text { No serious } \\
\text { imprecision }\end{array}$ & None & 404 & 408 & - & MD 0.45 higher ( 0.12 lower to 1.03 higher) & $\begin{array}{l}\text { o++ooo Very } \\
\text { low }\end{array}$ & Important \\
\hline \multicolumn{13}{|c|}{ FSH (follow-up mean 3 months; better indicated by lower values) } \\
\hline 9 & Randomized trial & Very serious & Serious & $\begin{array}{l}\text { No serious } \\
\text { indirectness }\end{array}$ & $\begin{array}{l}\text { No serious } \\
\text { imprecision }\end{array}$ & Reporting bias & 429 & 433 & - & SMD 0.09 lower ( 0.23 lower to 0.04 higher) & $\begin{array}{c}\text { o++ooo Very } \\
\text { low }\end{array}$ & Important \\
\hline \multicolumn{13}{|c|}{$E_{2}$ (follow-up mean 3 months; better indicated by lower values) } \\
\hline 9 & Randomized trial & Very serious & Serious & $\begin{array}{l}\text { No serious } \\
\text { indirectness }\end{array}$ & $\begin{array}{l}\text { No serious } \\
\text { imprecision }\end{array}$ & Reporting bias & 429 & 433 & - & MD 5.19 higher (2.06 to 8.33 higher) & $\begin{array}{c}\text { o++ooo Very } \\
\text { low }\end{array}$ & Important \\
\hline \multicolumn{13}{|c|}{ P (follow-up mean 3 months; better indicated by lower values) } \\
\hline 5 & Randomized trial & Very serious & Serious & $\begin{array}{l}\text { No serious } \\
\text { indirectness }\end{array}$ & $\begin{array}{l}\text { No serious } \\
\text { imprecision }\end{array}$ & Reporting bias & 286 & 289 & - & MD 0.27 higher ( 0.07 to 0.46 higher) & $\begin{array}{c}\text { o++ooo Very } \\
\text { low }\end{array}$ & Important \\
\hline
\end{tabular}


treatment of oligomenorrhea. This research, however, has some limitations due to the lack of alternative herbal medicine reference from China (3). In the present study, we performed the first comprehensive SR evaluating the efficacy and safety of TCM on treating oligomenorrhea so far, presenting an important strength. Our research demonstrates the potential feasibility of TCM as a kind of complementary and alternative medicine for the effective treatment of oligomenorrhea. The finding can be further applied to clinical practice to guide TCM and modern medicine practitioners to choose medicines with better effects and fewer side effects. Our findings have provided evidence for the future update of TCM and integrative medicine treatment guidelines of oligomenorrhea.

Limitations of this systemic review and meta-analysis should be acknowledged. First, different intervention settings (e.g., a wide spectrum of dosages, dosage forms, and ingredients of Chinese herbal medicines have been used in prior studies) and control group settings in prior studies caused inevitable heterogeneity. To deal with the heterogeneity, the random-effect model was applied in our analysis. Second, the methodological quality of the included studies was generally poor. To be specific, twenty-one studies only mentioned random allocation but failed to provide the details in randomization. No trial mentioned allocation concealment or blinding of participants and medical personnel. Moreover, all the included studies designed a blank control group, but none of them used placebo control. These flaws may lead to an exaggeration of experimental results. As for publication bias, only the outcome of effectiveness rates did not display a great significance. As a result, potential publication bias cannot be ruled out. Furthermore, all the included studies were conducted based on China population. It may preclude the generalizability of current evidence to other demographic groups.

\section{Conclusions}

In conclusion, evidence from our meta-analysis indicates that TCMs plus BM might be more efficacious as a preferred therapy to treat oligomenorrhea than $\mathrm{BM}$ alone. The potential contributions TCMs make include increasing menstrual blood volumes, prolonging menstrual periods, increasing endometrial thickness, and increasing the levels of $\mathrm{E}_{2}$ and $\mathrm{P}$.

However, current evidence has been hampered by the fact that the included trials are generally of low methodological quality. Current findings need to be validated via further RCTs with rigorous designs and in diverse populations if possible.

\section{Acknowledgments}

Funding: This study was supported by grant 81674014 and 81873330 from the National Natural Science Foundation of China, and grant 2017G006017 from the Key Research and Development Project of Shandong Province.

\section{Footnote}

Provenance and Peer Review: This article was commissioned by the editorial office, Annals of Palliative Medicine for the series "Narrative \& Evidence-based Medicine for Traditional Medicine: from basic research to clinical practice and trial". The article has undergone external peer review.

Reporting Checklist: The authors have completed the PRISMA reporting checklist. Available at http://dx.doi. org/10.21037/apm-20-825

Conflicts of interest: All authors have completed the ICMJE uniform disclosure form (available at http://dx.doi. org/10.21037/apm-20-825). The series "Narrative \& Evidence-based Medicine for Traditional Medicine: from basic research to clinical practice and trial" was commissioned by the editorial office without any funding or sponsorship. BL served as the unpaid Guest Editor of the series. The authors have no other conflicts of interest to declare.

Ethical Statement: The authors are accountable for all aspects of the work in ensuring that questions related to the accuracy or integrity of any part of the work are appropriately investigated and resolved.

Open Access Statement: This is an Open Access article distributed in accordance with the Creative Commons Attribution-NonCommercial-NoDerivs 4.0 International License (CC BY-NC-ND 4.0), which permits the noncommercial replication and distribution of the article with the strict proviso that no changes or edits are made and the original work is properly cited (including links to both the formal publication through the relevant DOI and the license). See: https://creativecommons.org/licenses/by-nc-nd/4.0/.

\section{References}

1. Medicine CAoC. Guidelines for Diagnosis and Treatment of Common Diseases of Gynecology in Traditional Chinese Medicine. Beijing: China Press of Traditional 
Chinese Medicine, 2012.

2. Tan Y. Gynecology of Chinese Medicine (Second Edition). Beijing: China Press of Traditional Chinese Medicine, 2016.

3. Moini Jazani A, Hamdi K, Tansaz M, et al. Herbal Medicine for Oligomenorrhea and Amenorrhea: A Systematic Review of Ancient and Conventional Medicine. Biomed Res Int 2018;2018:3052768.

4. Munro MG, Critchley HOD, Fraser IS. The two FIGO systems for normal and abnormal uterine bleeding symptoms and classification of causes of abnormal uterine bleeding in the reproductive years: 2018 revisions. Int J Gynaecol Obstet 2018;143:393-408.

5. Izhar R, Husain S, Tahir S, et al. Occult Form of Premature Ovarian Insufficiency in Women with Infertility and Oligomenorrhea as Assessed by Poor Ovarian Response Criteria. J Reprod Infertil 2017;18:361-7.

6. Nezi M, Christopoulos P, Paltoglou G, et al. Focus on $\mathrm{BMI}$ and subclinical hypothyroidism in adolescent girls first examined for amenorrhea or oligomenorrhea. The emerging role of polycystic ovary syndrome. J Pediatr Endocrinol Metab 2016;29:693-702.

7. Harris HR, Babic A, Webb PM, et al. Polycystic Ovary Syndrome, Oligomenorrhea, and Risk of Ovarian Cancer Histotypes: Evidence from the Ovarian Cancer Association Consortium. Cancer Epidemiol Biomarkers Prev 2018;27:174-82.

8. Capozzi A, Scambia G, Pontecorvi A, et al. Hyperprolactinemia: pathophysiology and therapeutic approach. Gynecol Endocrinol 2015;31:506-10.

9. Ding L, Cao J, Hu C. Study on the symptoms of menstrual diseases caused by three commonly used contraceptives and the syndrome pattern of traditional Chinese medicine. Journal of Traditional Chinese Medicine 2011;52:61-2.

10. Lu Q, Lin J, Jin Z, et al. The relationship between oligomenorrhea and endometrial blood flow. Chinese Journal of Obstetrics and Gynecology 2012;47:152-3.

11. Wang Y, Shi W, Liu J. Research progress of traditional Chinese medicine in the treatment of oligomenorrhea after artificial abortion. Information on Traditional Chinese Medicine 2018;35:122-5.

12. Liu KE, Hartman M, Hartman A. Management of thin endometrium in assisted reproduction: a clinical practice guideline from the Canadian Fertility and Andrology Society. Reprod Biomed Online 2019;39:49-62.

13. Zhou X, Yang X. Advances in research on the relationship between obesity and menstrual abnormalities. Chinese Journal of Obstetrics and Gynecology 2018;53:581-4.
14. Zhu D, Geng H, Zhang P. The cause and the progress of treatment of hypomenorrhea. Chinese Journal of Reproductive Health 2017;28:597-600.

15. Zhai P, Gao Y. Progress in research on Chinese and Western medicine with hypomenorrhea. Modern Medical Journal 2015;43:119-22.

16. Glueck CJ, Woo JG, Khoury PR, et al. Adolescent oligomenorrhea (age 14-19) tracks into the third decade of life (age 20-28) and predicts increased cardiovascular risk factors and metabolic syndrome. Metabolism 2015;64:539-53.

17. Lai L, Flower A, Prescott P, et al. Standardised versus individualised multiherb Chinese herbal medicine for oligomenorrhoea and amenorrhoea in polycystic ovary syndrome: a randomised feasibility and pilot study in the UK. BMJ Open 2017;7:e011709.

18. Tang JL, Liu BY, Ma KW. Traditional Chinese medicine. Lancet 2008;372:1938-40.

19. Yao R, Liu C. Research status of traditional Chinese medicine in the treatment of oligomenorrhea. Clinical Journal of Traditional Chinese Medicine 2018;30:373-6.

20. Department of Gynecology and Endocrinology OaGB, Chinese Medical Association. Guideline for diagnosis and treatment of abnormal uterine bleeding. Chinese Journal of Obstetrics and Gynecology 2014;49:74-9.

21. Liberati A, Altman DG, Tetzlaff J, et al. The PRISMA statement for reporting systematic reviews and metaanalyses of studies that evaluate healthcare interventions: explanation and elaboration. BMJ 2009;339:b2700.

22. Higgins JP, Green S. Cochrane handbook for systematic reviews of interventions (Version 5.1.0). Available online: http://handbook-5-1.cochrane.org/. 2011.

23. Balshem H, Helfand M, Schunemann HJ, et al. GRADE guidelines: 3. Rating the quality of evidence. J Clin Epidemiol 2011;64:401-6.

24. Cai C. Clinical study on Jieyu Zishen Decoction combined with Bromocriptine in treating hypercoagulogenemia with liver depression and kidney deficiency [MD]: Fujian University of Traditional Chinese Medicine, 2018.

25. Cheng H, Niu H, Lan X, et al. Sequential treatment of Hexue Decoction and Yangjing Tongluo Decoction combined with estrogen and progesterone cycle treatment for 30 cases of hypomenorrhea after induced abortion. Journal of Anhui University of Chinese Medicine 2016;35:21-4.

26. Gao S. Effects of artificial cycle therapy plus biochemical decoction on 53 cases of hypomenorrhea after artificial abortion. Medical Journal of Communications 
2014;28:488-9.

27. Hei L. Observation on the therapeutic effects of Yangshen Huayu Recipe on treatment of hypomenorrhea caused by inferior endometrium. Shaanxi Journal of Traditional Chinese Medicine 2017;38:1085-6.

28. Hu X, Xu Y, Liu Y, et al. Effects of modified Guishen Pill combined with estrogen and progesterone artificial cycle on the treatment of hypomenorrhea with kidney deficiency and blood stasis after artificial abortion. Modern Journal of Integrated Traditional Chinese and Western Medicine 2017;26:819-21.

29. Li J, Ou Y. Clinical efficacy of Dingkun Pills combined with hormone in the treatment of patients with hypomenorrhea with kidney deficiency and liver stagnation syndrome and effects on endometrial thickness. World Chinese Medicine 2018;13:669-71+75.

30. Li Y, Huang J, huang L, et al. Treatment of 150 cases of hypomenorrhea after abortion. Chinese Community Doctors 2012;14:225.

31. Li Q. Clinical observation on the treatment of hypomenorrhea after painless abortion of kidney deficiency and blood stasis type by combination of traditional Chinese medicine and western medicine. Hubei Journal of Traditional Chinese Medicine 2011;33:38-9.

32. Lu K, Ma Z, Ban S, et al. Maixuekang capsule combined with estrogen and progesterone for the treatment of hypomenorrhea with blood stasis type and unclear reasons. Chinese Traditional Patent Medicine 2017;39:1582-5.

33. Lu Y. Therapeutic effects of combination of traditional Chinese and western medicine on postoperative hypomenorrhea after artificial abortion. Modern Journal of Integrated Traditional Chinese and Western Medicine 2016;25:197-8.

34. Ma X, Tang W, Suo L, et al. Clinical observation on treatment of hypomenorrhea with decreased ovarian reserve function by Bushen Huoxue decoction combined with kelingen. Zhejiang Clinical Medical Journal 2017;19:253-5.

35. Ruan R. Clinical observation on the treatment of hypomenorrhea after abortion with integrated traditional Chinese and Western medicine. Modern Journal of Integrated Traditional Chinese and Western Medicine 2012;21:1174-5.

36. Song J, Lan F. Clinical observation on Kunling Pill combined with estrogen and progesterone sequential therapy for hypomenorrhea after abortion. China Practical Medicine 2011;6:138-9.

37. Tang C, Wang A, Yan Q. Effects of Bushen Jianpi Yangxue
Ointment on the treatment of hypomenorrhea with kidney deficiency and blood stasis type and its effect on endometrial thickness and sex hormone. Shanxi Journal of Traditional Chinese Medicine 2018;39:1333-5.

38. Tian B. Evaluation on the therapeutic effects of Bushen Zixue Recipe combined with Kelingong on hypomenorrhea with kidney and blood deficiency type after artificial abortion [MD]: Henan Universiyt of Chinese Medicine, 2016.

39. Wang Q. Clinical observation on the treatment of hypomenorrhea after abortion with integrated traditional Chinese and Western medicine. Medical Information 2014;27:282.

40. Wang Y. Clinical observation on the treatment of hypomenorrhea with liver and kidney deficiency type by Yangjing Zixue Decoction and Bujiale [MD]: Shandong University of Traditional Chinese Medicine, 2016.

41. Wang Z. Simultaneous treatment of Siwu Decoction and Western medicine artificial cycle therapy in the treatment of hypomenorrhea. China's Naturopathy 2015;23:70-1.

42. Xiong J, Du H. Clinical observation on 66 cases of hypomenorrhea after abortion treated with estradiol valerate and progesterone capsule combined with Bushen Huoxue Decoction. Modern Diagnosis and Treatment 2017;28:2790-1.

43. Xue X. Therapeutic effects of Huoxue Tiaojing Pills on the treatment of hypomenorrhea after artificial abortion. Journal of Practical Gynecologic Endocrinology 2015;2:31-2.

44. Zhang F. Clinical observation on the treatment of hypomenorrhea after painless artificial abortion with traditional Chinese medicine combined with western medicine. Journal of New Chinese Medicine 2015;47:143-4.

45. Zhang H, Hong C, Liu C. Therapeutic effects of traditional Chinese medicine sequential therapy combined with Climen on hypomenorrhea after artificial abortion. Journal of Anhui University of Chinese Medicine 2018;37:15-8.

46. Zheng Y, Zhao Y, Luo S. Observation on the therapeutic effect of Zishen Yutai Pill in treating hypomenorrhea with kidney deficiency. Journal of Chinese Medicinal Materials 2015;38:203-5.

47. Zhang $Z$. Therapeutic effects of integrated traditional Chinese and western medicine on patients with hypomenorrhea after abortion. Cardiovascular Disease Journal of Integrated Traditional Chinese and Western Medicine 2016;4:180. 
48. Zhao J. Clinical observation on the treatment of hypomenorrhea after abortion with integrated traditional Chinese and Western medicine. Chinese Community Doctors 2014;30:129-30.

49. Zheng $Z$. Treatment of 63 cases of hypomenorrhea after artificial abortion with Huoxue Tiaojing Pill. China Pharmaceuticals 2015;24:92-3.

50. Su JY, Xie QF, Liu WJ, et al. Perimenopause Amelioration of a TCM Recipe Composed of Radix Astragali, Radix Angelicae Sinensis, and Folium Epimedii: An In Vivo Study on Natural Aging Rat Model. Evid Based Complement Alternat Med 2013;2013:747240.

51. Xu Y, Li X, Chen T, et al. Radix Paeoniae Alba increases serum estrogen level and up-regulates estrogen receptor expression in uterus and vagina of immature/ ovariectomized mice. Phytother Res 2019;33:117-29.

52. Bi X, Han L, Qu T, et al. Anti-Inflammatory Effects, SAR, and Action Mechanism of Monoterpenoids from Radix Paeoniae Alba on LPS-Stimulated RAW 264.7 Cells. Molecules 2017;22:715.

53. Zhou Y, Tao H, Wang A, et al. Chinese herb pair Paeoniae Radix Alba and Atractylodis Macrocephalae Rhizoma suppresses LPS-induced inflammatory response through inhibiting MAPK and NF-kappaB pathway. Chin Med 2019;14:2.

54. Du K, Gao X, Wang F, et al. Research progress on quality and efficacy evaluation of Rehmanniae Radix Praeparata based on pharmacodynamic material basis. Chinese Traditional and Herbal Drugs 2019;50:1477-84.

55. Li N. Research progress on pharmacological effects of the active ingredients of Radix Rehmanniae Preparata. Journal of China Prescription Drug 2017;15:14-5.

56. Ke J, Duan R. Effects of flavonoids from semen cuscutae on the hippocampal-hypothalamic-pituitary-ovarian sex hormone receptors in female rats exposed to psychological stress. Clin Exp Obstet Gynecol 2013;40:271-4.

57. Ding JX, Li WL, Hu Y, et al. Characterization of estrogenic active ingredients in Cuscuta chinensis Lam. based on spectral characteristics and highperformance liquid chromatography/quadrupole timeofflight mass spectrometry. Mol Med Rep 2019;19:1238-47.

58. Miao M, Peng M, Zhu Z, et al. Effects of dodder total flavone on polycystic ovary syndrome rat models induced by DHEA combined HCG. Saudi J Biol Sci 2019;26:821-7.

59. Yang S, Xu X, Xu H, et al. Purification, characterization and biological effect of reversing the kidney-yang deficiency of polysaccharides from semen cuscutae. Carbohydr Polym
2017;175:249-56.

60. Gu J, Su S, Guo J, et al. Anti-inflammatory and antiapoptotic effects of the combination of Ligusticum chuanxiong and Radix Paeoniae against focal cerebral ischaemia via TLR4/MyD88/MAPK/NF-kappaB signalling pathway in MCAO rats. J Pharm Pharmacol 2018;70:268-77.

61. Lu J, Chen X, Xu X, et al. Active polypeptides from Hirudo inhibit endothelial cell inflammation and macrophage foam cell formation by regulating the LOX1/LXR-alpha/ABCA1 pathway. Biomed Pharmacother 2019;115:108840.

62. Zhang X, Zheng W, Wang T, et al. Danshen-ChuanxiongHonghua Ameliorates Cerebral Impairment and Improves Spatial Cognitive Deficits after Transient Focal Ischemia and Identification of Active Compounds. Front Pharmacol 2017;8:452.

63. Wang Y, Guo G, Yang BR, et al. Synergistic effects of Chuanxiong-Chishao herb-pair on promoting angiogenesis at network pharmacological and pharmacodynamic levels. Chin J Integr Med 2017;23:654-62.

64. Jin Y. Protective effects of achyranthes bidentata polysaccharides on acute liver injury induced by carbon tetrachloride in rats. Journal of Medical Science Yanbian University 2018;41:248-51.

65. Tao JH, Zhao M, Jiang S, et al. UPLC-Q-TOF/MS-based metabolic profiling comparison of four major bioactive components in normal and CKD rat plasma, urine and feces following oral administration of Cornus officinalis Sieb and Rehmannia glutinosa Libosch herb couple extract. J Pharm Biomed Anal 2018;161:254-61.

66. Liu YM, Zhu LL, Li R, et al. Xijiao Dihuang Decoction () and Rehmannia glutinosa Libosch. protect mice against lipopolysaccharide and tumor necrosis factor alpha-induced acute liver failure. Chin J Integr Med 2019;25:446-53.

67. Mo ZZ, Liu YH, Li CL, et al. Protective Effect of SFE$\mathrm{CO} 2$ of Ligusticum chuanxiong Hort Against d-GalactoseInduced Injury in the Mouse Liver and Kidney. Rejuvenation Res 2017;20:231-43.

Cite this article as: $\mathrm{Li} \mathrm{Y}$, Zhao G, Shi W, Zhang Y, Diao H, Ding N, Li P, Zhang F, Yang L, Sun M, Yu H, Li B, Xu L. Efficacy and safety of traditional Chinese medicine on treating oligomenorrhea: a systematic review and meta-analysis. Ann Palliat Med 2021;10(12):12955-12968. doi: 10.21037/apm-20-825 


\section{Supplementary}

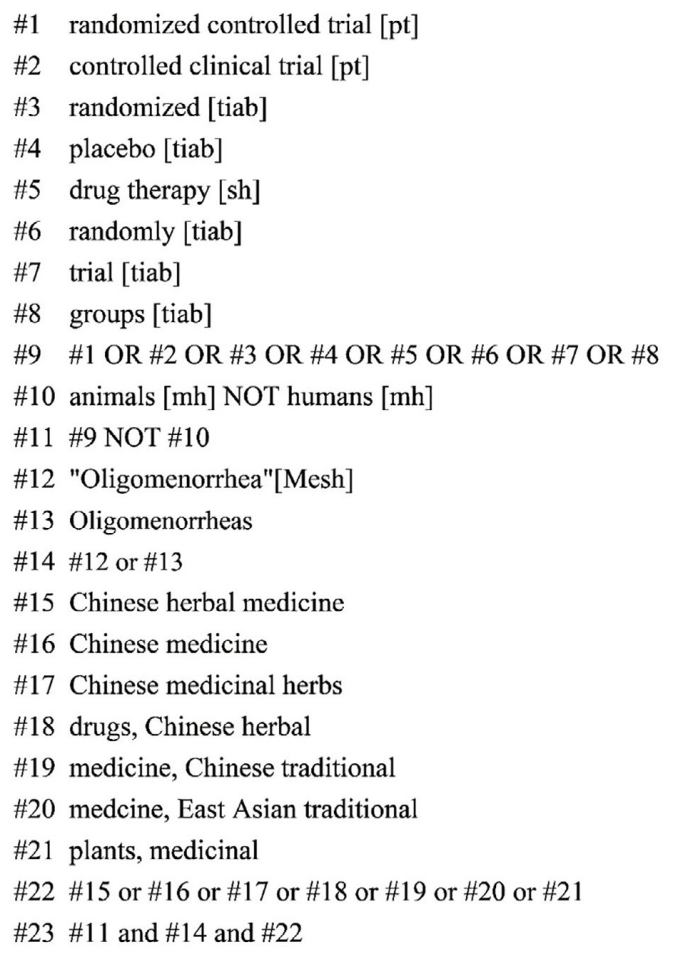

Figure S1 Search Strategy.

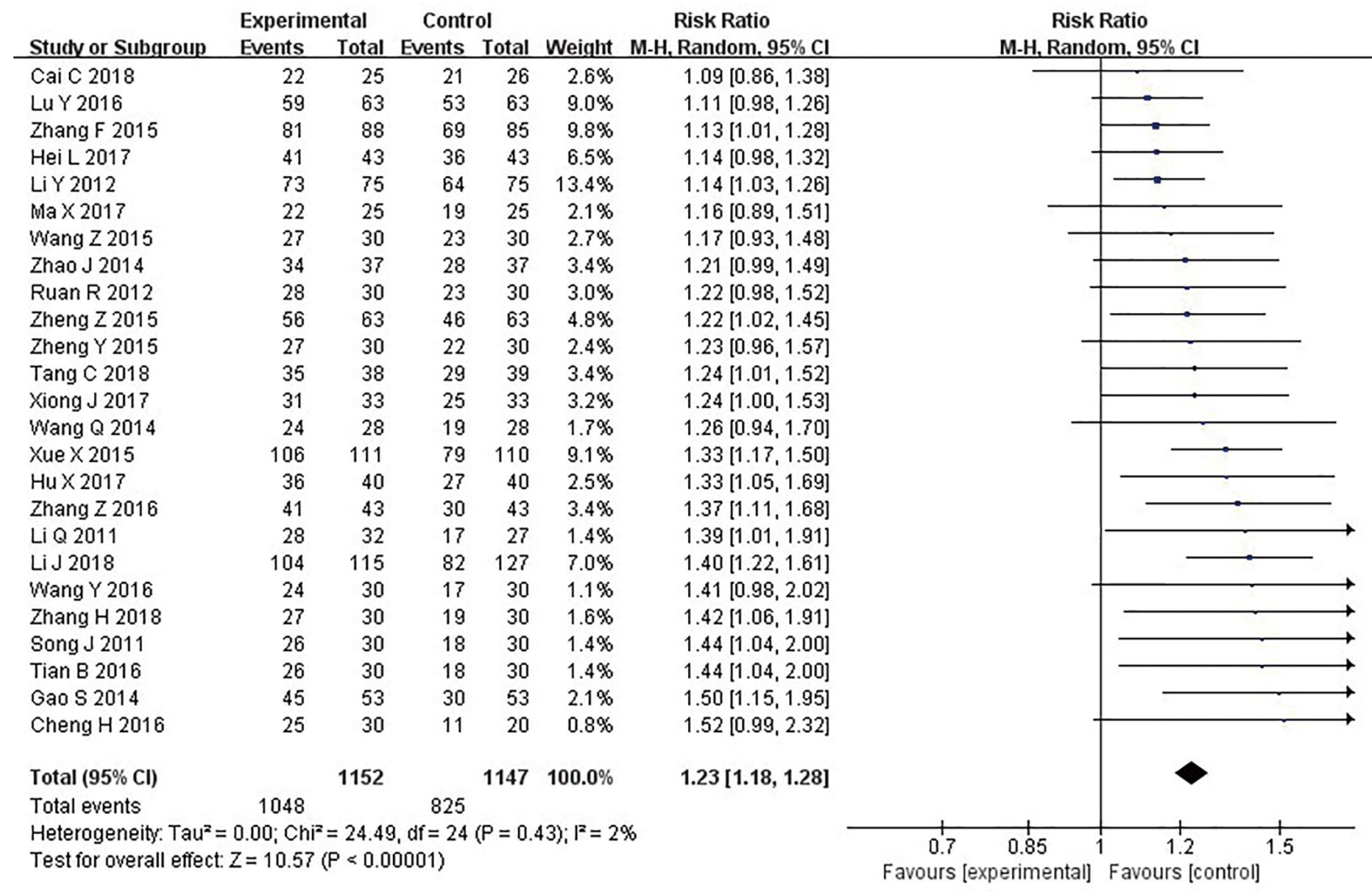

Figure S2 meta-analysis results of TCM plus BM versus BM alone in terms of effective rate. 


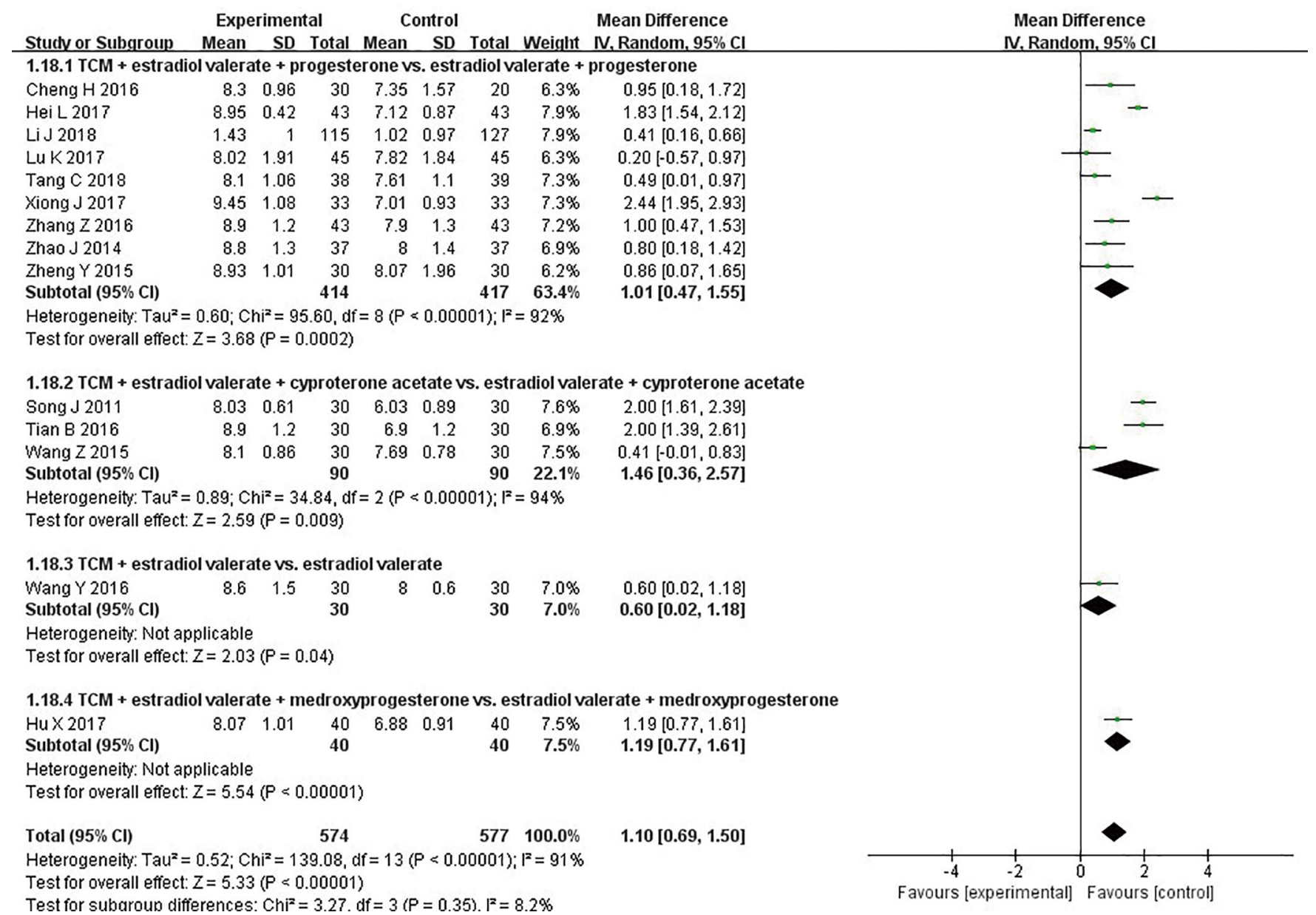

Figure S3 meta-analysis results of TCM plus BM versus BM alone in terms of endometrial thickness.

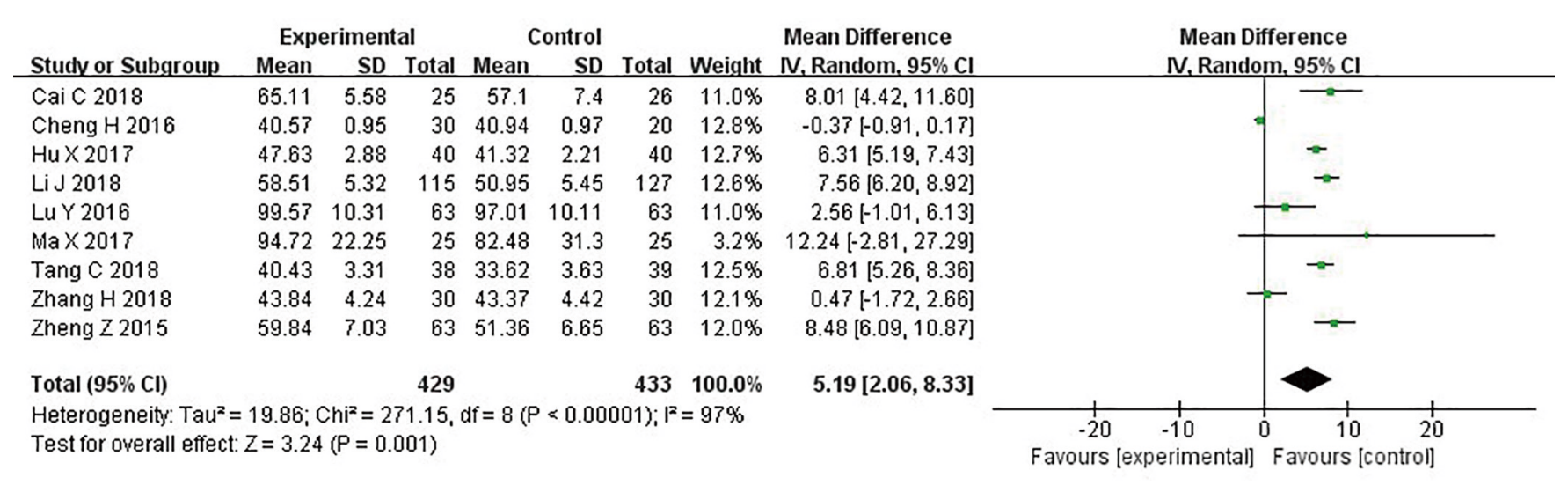

Figure S4 meta-analysis results of TCM plus BM versus BM alone in terms of $\mathrm{E}_{2}$. 


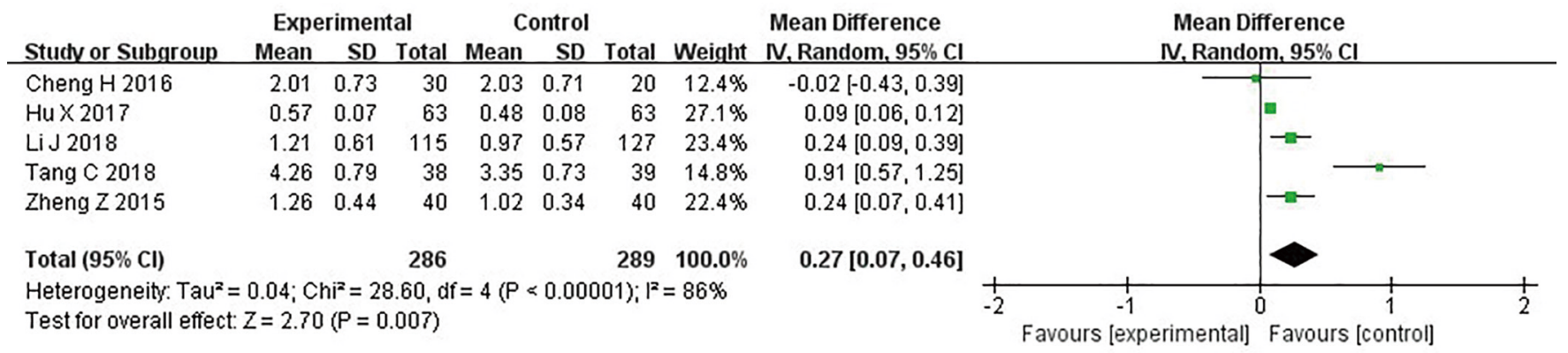

Figure S5 meta-analysis results of TCM plus BM versus BM alone in terms of $\mathrm{P}$.

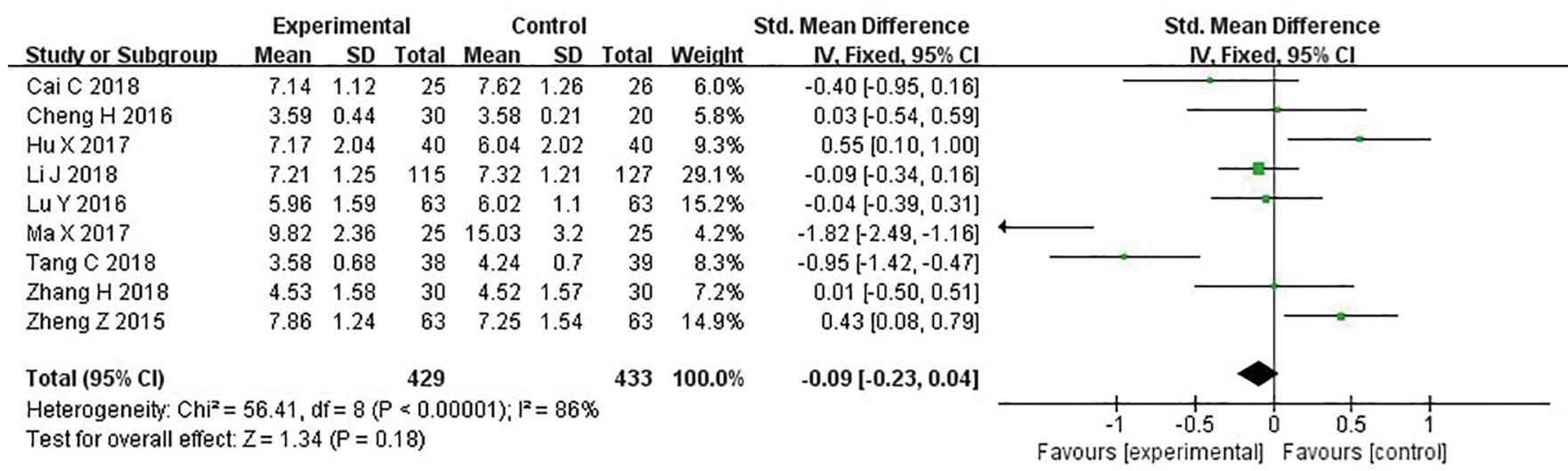

Figure S6 meta-analysis results of TCM plus BM versus BM alone in terms of FSH.

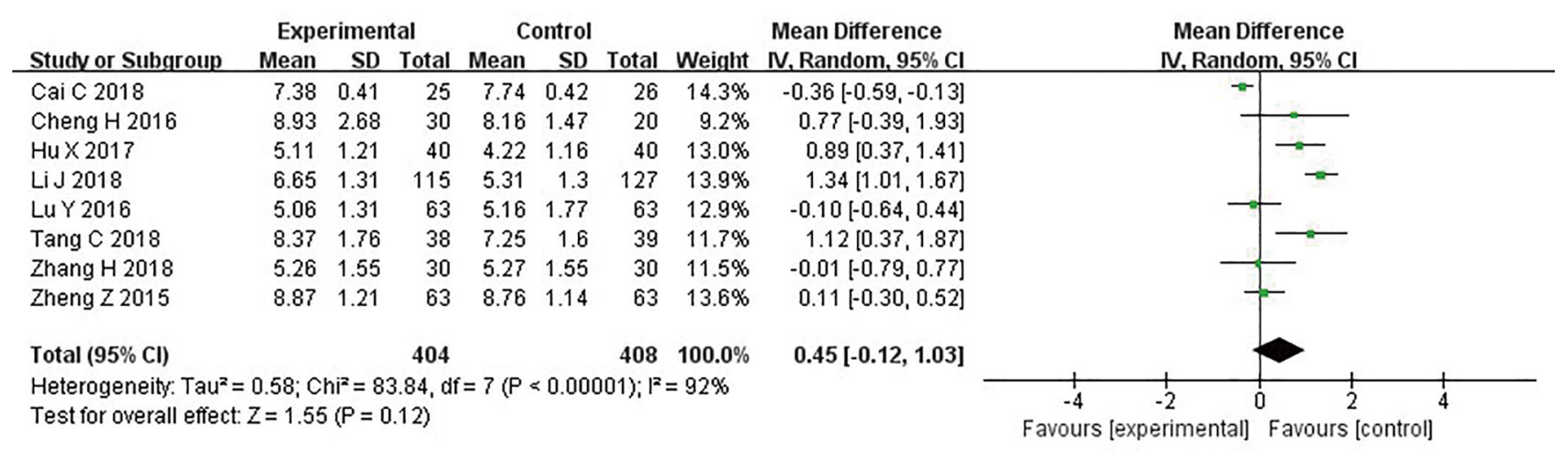

Figure S7 meta-analysis results of TCM plus BM versus BM alone in terms of LH. 
Table S1 Components of the included TCM nourishing blood circulation

Bushen Yangjing
Decoction

Yangjing Tongluo Decoction

(2) Nourishing blood and promoting blood circulation

Gao S 2014 Nourishing blood and promoting blood circulation

Hei L 2017

Tonifying the kidney and promoting blood circulation

Tonifying the kidney and promoting blood circulation

HuX 2017

Shenghua Decoction

Yangshen Huayu

Decoction

Guishen Decoction

Dingkun Pill

Tonifying the kidney and promoting blood circulation

Soothing the liver and nourishing blood

Decoction

Promoting blood circulation, promoting qi an warming wo

Promoting blood circulation, promoting giand

Invigorating the kidney and the spleen reinforcing qi and nourishing blood

Tonifying the kidney and promoting blood circulation

Tonifying the kidney and promoting blood circulation

Invigorating the kidney and the spleen nourishing essence and blood

Invigorating the kidney, nourishing blood,

nvigorating the kidney and the spleen promoting blood circulation and nourishing promo
blood

Tonifying the kidney and promoting blood circulation

Decoction for intermenstrual period Decoction for post menstrual period Maixuekang Capsule Self-made Decoction

Bushen Huoxue Decoctio

Jianpi Bushen Yijing Yangxue Decoctio

Kunling Pill

Bushen Jianpi Yangxue Ointment

Bushen Zixue Decoction

Siwu Decoction

Yangjing Zixue Decoction

Siwu Decoction

Bushen Huoxue Decoction Huoxue Tiaojing Pil

Reinforcing ai, nourishing blood and removing Chanfukang Granule

Zhang F 2015

Zhang $\mathrm{H} 2018$

Tonifying the kidney and nourishing blood

Tonifying the kidney and supporing Yang

Promoting blood circulation and removing blood stasis

Tonifying the kidney Invigorating the kidney and the spleen, and
nourishing blood

Zhang Z 2016

Zhao J 2014 nourishing blood

Decoction for post

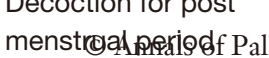

Decoction for menstrual

period Yangxue Decoction

Bushen Jianpi Yijing
Yangxue Decoction
Components

Radix Bupleuri (chai hu), Radix Paeoniae Alba (bai shao), Radix Angelicae Sinensis (dang gui), Fructus Hordei Germinatus (mai ya), Semen Cuscutae (tu si zi), Fructus Lycii (gou qi zi), Radix

Rehmanniae Preparata (shu di huang), Rhizoma Cyperi (xiang fu), Poria (fu ling), Radix Glycyrrhizae (zhi gan cao)

Semen Cuscutae (tu si zi), Radix Rehmanniae Preparata (shu di huang), Rhizoma Dioscoreae (shan yao), Fructus Corni (shan zhu yu), Radix Paeoniae Alba(bai shao), Radix Pseudostellariae (tai zi shen), Radix Ophiopogonis (mai dong), Radix Astragali seu Hedysari (huang qi), Radix Angelicae Sinensis (dang gui), Rhizoma Ligustici Chuanxiong (chuan xiong), Radix Paeoniae Rubra (chi shao), Semen Persicae (tao ren), Flos Carthami (hong hua), Radix Glycyrrhizae (zhi gan cao)

Herba Leonuri (yi mu cao), Radix Cyathulae (chuan niu xi), Radix Angelicae Sinensis (dang gui), Rhizoma Ligustici Chuanxiong (chuan xiong), Radix Paeoniae Rubra (chi shao), Rhizoma Sparganii (san leng), Rhizoma Curcumae (e zhu), Rhizoma Cyperi (xiang fu), Flos Rosae Chinensis (yue ji hua)

Radix Angelicae Sinensis (dang gui), Rhizoma Ligustici Chuanxiong (chuan xiong), Semen Persicae (tao ren), Rhizoma Zingiberis (gan jiang), Radix Glycyrrhizae (zhi gan cao)

Radix Paeoniae Alba(bai shao), Radix Rehmanniae Preparata (shu di huang), Fructus Hordei Germinatus (sheng mai ya), Radix Angelicae Sinensis (dang gui). Cortex Eucommiae (du zhong), Radix Dipsaci (xu duan), Semen Persicae (tao ren), Ramulus Cinnamomi (gui zhi), Radix Glycyrrhizae (zhi gan cao), Rhizoma Ligustici Chuanxiong (chuan xiong)

Radix Rehmanniae Preparata (shu di huang), Rhizoma Dioscoreae (shan yao), Semen Cuscutae (tu si zi), Cortex Eucommiae (du zhong), Fructus Lycii (gou qi zi), Poria (ful ling), Radix Angelicae Sinensis (dang gui), Rhizoma Ligustici Chuanxiong (chuan xiong), Caulis Spatholobi (ii xue teng), Radix Cyathulae (chuan niu xi), Radix Glycyrrhizae (sheng gan cao)

Radix Ginseng Rubra (hong shen), Cornu Cervi Pantotrichum (lu rong), Stigma Croci (xi hong hua), Radix Notoginseng (san qi), Radix Paeoniae Alba(bai shao), Radix Rehmanniae Preparata (shu di huang), Radix Angelicae Sinensis (dang gui), Rhizoma Atractylodis Macrocephalae (bai zhu), Fructus Lycii (gou qi zi), Radix Scutellariae (huang qin), Rhizoma Cyperi (xiang fu), Fructus Leonuri (chong wei zi), Rhizoma Ligustici Chuanxiong (chuan xiong), Cornu Cervi Degelatinatum (lu jlao shuang), Colla Corii Asini (e jlao), Rhizoma Corydalis (yan hu suo), Caulis Spatholobi (ii xue teng), Flos Carthami (hong hua), Herba Leonuri (yi mu cao), Faeces Togopteri (wu ling zhi), Poria (fu ling), Radix Bupleuri (Chai Hu), Radix Linderae (wu yao), Fructus Amomi Villosi (sha ren), Cortex Eucommiae (du zhong), Rhizoma Zingiberis (gan jiang), Asarum sieboldii Miq (xi xin), Radix Cyathulae (chuan niu xi), Cortex Cinnamomi (rou gui), Radix Glycyrrhizae (zhi gan cao)

Semen Cuscutae (tu si zi), Fructus Ligustri Lucidi (nv zhen zi), Flos Carthami (hong hua), Radix Rehmanniae Preparata (shu di huang), Radix Codonopsis (dang shen), Cistanche deserticola Ma (rou
cong rong), Radix Morindae Officinalis (ba ji tian), Rhizoma Curcumae Longae (iiang huang), Flos Rosae Chinensis (yue ji hua), Cortex Moutan Radicis (mu dan pi), Radix Rubiae (qian cao), Radix Glycyrhizae (sheng gan cao)

Cornu cervi (lu jiao), Radix Morindae Officinalis (ba ji tian), Trigonella foenum-graecum L. (hu lu ba), Herba Cynomorii (suo yang), Radix Rehmanniae Preparata (shu di huang), Radix Angelicae Sinensis (z) gui), Radix Paeoniae Alba(bai shao), Fructus Ligustri Lucidi (nv zhen zi), Fructus Lycii (gou qi zi), Radix Glycyrrhizae (zhi gan cao)

Rhizoma Sparganii (san leng), Rhizoma Curcumae (e zhu), Rhizoma Cyperi (xiang fu), Radix Achyranthis Bidentatae (niu xi), Radix Angelicae Sinensis (dang gui), Citrus aurantium L. (zhi qiao), Rhizoma Ligustici Chuanxiong (chuan xiong), Flos Carthami (hong hua), Citrus reticulata Blanco (iv ye), Semen Citri Reticulatae (iv he), Flos Rosae Chinensis (yue ji hua), Flos Rosae Rugosae (mei gui hua),

Radix Angelicae Sinensis (dang gui), Flos Carthami (hong hua), Rhizoma Ligustici Chuanxiong (chuan xiong), Rhizoma Cyperi (xiang fu), Rhizoma Alismatis (ze xie), Cornu cervi (lu jiao), Radix Morindae Officinalis (ba ji tian), Trigonella foenum-graecum L. (hu lu ba), Herba Cynomorii (suo yang), Ramulus Cinnamomi (gui zhi), Radix Puerariae (ge gen)

Radix Rehmanniae Preparata (shu di huang), Fructus Corni (shan zhu yu), Rhizoma Dioscoreae (shan yao), Radix Salviae Miltiorrhizae (dan shen), Fructus Ligustri Lucidi (nv zhen zi), Herba Ecliptae (mo han lian), Radix Angelicae Sinensis (dang gui), Radix Paeoniae Alba(bai shao), Radix Astragali seu Hedysari (huang qi), Radix Codonopsis (dang shen), Radix Glycyrrhizae (zhi gan cao) Hirudo (shui zhi)

Poria (fu ling), Rhizoma Dioscoreae (shan yao), Fructus Corni (shan zhu yu), Cortex Eucommie (du zhong). Radix Rehmanniae Preparata (shu di huang). Fructus Lycii (gou gi zi), Placenta Hominis (z he che), Semen Cuscutae (tu si zi), Radix Paeoniae Rubra (chi shao), Radix Angelicae Sinensis (dang gui)

Semen Cuscutae (tu si zi), Cistanche deserticola Ma (rou cong rong), Radix Angelicae Sinensis (dang gui), Rhizoma Ligustici Chuanxiong (chuan xiong), Rhizoma Dioscoreae (shan yao), Radix Bupleuri (Chai Hu), Radix Paeoniae Alba(bai shao), Radix Rehmanniae Recens (sheng di huang)

Radix Rehmanniae Preparata (shu di huang), Fructus Lycii (gou qi zi), Semen Cuscutae (tu si zi), Rhizoma Atractylodis Macrocephalae (bai zhu), Semen Nelumbinis (lian zi), Cornu Cervi Degelatinatum (lu jiao shuang), Radix Paeoniae Alba(bai shao), Poria (fu ling), Rhizoma Dioscoreae (shan yao), Fructus Corni (shan zhu yu), Radix Angelicae Sinensis (dang gui), Herba Epimedii (yin yang huo), Fluoritum (z shi ying)

Rhizoma Cyperi (xiang fu), Herba Leonuri (yi mu cao), Flos Carthami (hong hua), Flos Celosiae Cristatae (ii guan hua), Radix Rehmanniae Recens (di huang), Radix Ophiopogonis (mai dong), Radix Paeoniae Alba(bai shao), Radix Astragali seu Hedysari (huang qi), Cistanche deserticola Ma (rou cong rong), Poria (fu ling), Cortex Magnoliae Officinalis (hou pu), Rhizoma Atractylodis Macrocephalae (bai zhu), etc

Radix Astragali seu Hedysari (huang qi), Radix Codonopsis (dang shen), Rhizoma Atractylodis Macrocephalae (bai zhu), Radix Angelicae Sinensis (dang gui), Radix Rehmanniae Preparata (shu di huang), Radix Paeoniae Alba(bai shao), Rhizoma Dioscoreae (shan yao), Fructus Corni (shan zhu yu), Semen Cuscutae (tu si zi), Fructus Lycii (gou qi zi), Poria (ful ling), Cortex Euco (i Mustri Lucidi (nv zhen zi), Herba Ecliptae (au) Radix Fructus Ligustri Lucidi (nv zhen zi), Herba Ecliptae (mo han lian), Pericarpium Citri Reticulatae (chen pi), Rhizoma Pinelliae (ban xia), Herba Epimedii (yin yang huo), Herba Leonuri (yi mu cao),
Cyathulae (chuan niu xi), Radix Morindae Officinalis (ba ji tian), Caulis Spatholobi (ji xue teng), Colla Corii Asini (e jiao), Radix Salviae Miltiorrhizae (dan shen), Fructus Amomi Villosi (sha ren)

Radix Rehmanniae Preparata (shu di huang), Fructus Lycii (gou qi zi), Fructus Corni (shan zhu yu), Rhizoma Polygonati (huang jing), Radix Angelicae Sinensis (dang gui), Radix Paeoniae Alba(bai shao), Rhizoma Atractylodis Macrocephalae (bai zhu), Rhizoma Ligustici Chuanxiong (chuan xiong), Rhizoma Cyperi (xiang fu), Radix Glycyrrhizae (gan cao)

Radix Rehmanniae Preparata (shu di huang), Radix Paeoniae Alba(bai shao), Radix Angelicae Sinensis (dang gui), Rhizoma Ligustici Chuanxiong (chuan xiong), Rhizoma Atractylodis Macrocephalae (bai zhu), Rhizoma Dioscoreae (shan yao), Pericarpium Citri Reticulatae (chen pi)

Cornu cervi (lu jiao), Radix Angelicae Sinensis (dang gui), Fructus Corni (shan zhu yu), Radix Paeoniae Alba(bai shao), Radix Rehmanniae Preparata (shu di huang), Fructus Mori (sang shen), Radix Polygoni Multiflori (he shou wu), Radix Salviae Miltiorrhizae (dan shen), Radix Puerariae (ge gen), Cortex Cinnamomi (rou gui), Radix Paeoniae Rubra (chi shao), Rhizoma Cyperi (xiang fu), Radix Cyathulae (chuan niu xi)

Radix Rehmanniae Preparata (shu di huang), Radix Paeoniae Alba(bai shao), Radix Angelicae Sinensis (dang gui), Rhizoma Ligustici Chuanxiong (chuan xiong), Radix Bupleuri (Chai Hu), Cortex Eucommiae (du zhong), Rhizoma Cyperi (xiang fu), Radix Salviae Miltiorrhizae (dan shen), Fructus Corni (shan zhu yu), Radix Morindae Officinalis (ba ji tian), Poria (fu ling), Rhizoma Dioscoreae (shan yao)

Caulis Spatholobi (ii xue teng), Radix Rehmanniae Preparata (shu di huang), Placenta Hominis (zi he che), Radix Angelicae Sinensis (dang gui), Radix Salviae Miltiorrhizae (dan shen), Rhizoma Cyper (xiang fu), Rhizoma Ligustici

Radix Rehmanniae Preparata (shu di huang), Radix Angelicae Sinensis (dang gui), Faeces Togopteri (wu ling zhi), Rhizoma Corydalis (yan hu suo), Radix Scutellariae (huang qin), Rhizoma Zingiberis (gan jiang), Radix Rehmanniae Recens (di huang), Pericarpium Citri Reticulatae Viride (qing pi), Pericarpium Citri Reticulatae (chen pi), Rhizoma Ligustici Chuanxiong (chuan xiong), Citrus aurantium L. Moutan Radicis (mu dan pi)

Herba Leonuri (yi mu cao), Radix Angelicae Sinensis (dang gui), Radix Ginseng (ren shen), Radix Astragali seu Hedysari (huang qi), Radix Polygoni Multiflori (he shou wu), Semen Persicae (tao ren) Typha angustifolia L (pu huang), Radix Rehmanniae Preparata (shu di huang), Rhizoma Cyperi (xiang fu), Thallus Laminariae (kun bu), Rhizoma Atractylodis Macrocephalae (bai zhu) Radix Rehmanniae Preparata (shu di huang), Caulis Spatholobi (ji xue teng), Semen Cuscutae (tu si zi), Fructus Lycii (gou qi zi), Fructus Corni (shan zhu yu), Rhizoma Dioscoreae (shan yao), Cortex

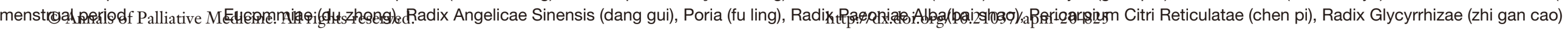
Semen Cuscutae (tu si zi), Herba Epimedii (yin yang huo), Cistanche deserticola Ma (rou cong rong), Radix Rehmanniae Preparata (shu di huang), Rhizoma Dioscoreae (shan yao), Fructus Corni (shan zhu yu), Cortex Eucommiae (du zhong), Fructus Rubi (fu pen zi), Radix Angelicae Sinensis (dang gui), Poria (fu ling) Semen Persicae (tao ren), Flos Carthami (hong hua), Radix Angelicae Sinensis (dang gui), Rhizoma Ligustici Chuanxiong (chuan xiong), Radix Paeoniae Rubra (chi shao), Radix Cyathulae (chuan niu xi), Caulis Spatholobi (ii xue teng), Rhizoma Cyperi (xiang fu), Herba Leonuri (yi mu cao)

Semen Cuscutae (tu si zi), Fructus Amomi Villosi (sha ren), Radix Rehmanniae Preparata (shu di huang), Radix Ginseng (ren shen), Herba Taxilli (sang ji sheng), Colla Corii Asini (e jiao), Radix Polygoni Multiflori (he shou wu), Folium Artemisiae Argyi (ai ye), Radix Morindae Officinalis (baji itian), Rhizoma Atractylodis Macrocephalo (bai zhu) Radix Codonopsis (dang shen), Cornu Cevvi Degelatinatum (It jao shuang), Fructus Lycii (gou qi zi), Radix Dipsaci (xu duan), Cortex Eucommiae (du zhong)

Bushen Jianpi Yijing Radix Rehmanniae Preparata (shu di huang), Semen Cuscutae (tu si zi), Fructus Lycii (gou qi zi), Rhizoma Atractylodis Macrocephalae (bai zhu), Cornu Cervi Degelatinatum (lu jiao shuang), Radix Paeoniae Alba(bai shao), Semen Nelumbinis (lian zi), Poria (fu ling), Rhizoma Dioscoreae (shan yao), Fructus Corni (shan zhu yu), Radix Angelicae Sinensis (dang gul)

Fructus Lycii (gou qi zi), Radix Rehmanniae Preparata (shu di huang), Radix Paeoniae Alba(bai shao), Rhizoma Atractylodis Macrocephalae (bai zhu), Semen Cuscutae (tu si zi), Poria (ful ling), Fructus Corni (shan zhu yu), Rhizoma Dioscoreae (shan yao), Cornu Cervi Degelatinatum (lu jiao shuang), Semen Nelumbinis (lian zi), Radix Angelicae Sinensis (dang gui), Fluoritum (zi shi ying), Herba Epimedii (yin yang huo)

Radix Rehmanniae Preparata (shu di huang), Radix Angelicae Sinensis (dang gui), Faeces Togopteri (wu ling zhi), Rhizoma Corydalis (yan hu suo), Radix Scutellariae (huang qin), Radix Rehmanniae Recens (di huang), Pericarpium Citri Reticulatae Viride (qing pi), Pericarpium Citri Reticulatae (chen pi), Rhizoma Ligustici Chuanxiong (chuan xiong), Citrus aurantium L. (zhi qiao), Rhizoma Cyperi (xiang
fu), Radix Paeoniae Rubra (chi shao), Lignum Sappan (su mu), Colla Corii Asini (e jiao), Flos Carthami (hong hua), Poria (fu ling), Fructus Amomi Villosi (sha ren), Cortex Moutan Radicis (mu dan pi) 